\title{
Psychooptische Untersuchungen.
}

I.

Über den Zusammenhang zwischen dem Reizungsgesetze und dem Gesetze Weber-Fechner's.

Von

J. K. A. Wertheim Salomonson, und

Professor an der Universität

zu Amsterdam.
G. J. Sehonte,

Augenarzt

(Mit 10 Textfiguren.)

Von einem der Verfasser ist ein . "Reizungsgesetz" abgeleitet worden, welches den Zusammenhang zwischen der Grösse eines physiologischen Effekts nnd der Grösse des vorausgegangenen Reizes ausdrückt.

Dieses Gesetz wird mathematisch dargestellt in folgender Formel:

$$
\left.E=A\left[1-\varepsilon^{-B(R-s)}\right]^{1}\right) \text {. . . . . (1). }
$$

während die Umschreibung so lautet:

"Wenn ein Reiz auf ein reizbares Organ einwirkt, so ist der Effekt um so grösser, je grösser der Reiz ist. Die Zunahme des Effektes bei Zunahme des Reizes ist um so geringer, je grösser der Reiz wird. Der Effekt nähert sich schliesslich asymptotisch einem gewissen Maximum, das nicht übersehritten wird, wie sehr man den Reiz auch verstärkt. Der Unterschied zwischen der wirklichen Effektgrösse und dem Maximum nimmt in geometrischer Proportion ab, wenn der Reiz in arithmetischer Proportion zunimmt."

In der mathematischen Formel stellt $A$ das Maximum des Effektes $E$ dar; $R$ ist der Reiz, $c$ der untere Schwellenwert desselben. $B$ bezeichnet die Zuwachskonstante, da sie angibt, wie schnell der Effekt bei Vergrösserung des Reizes zunimmt.

1) J.K. A. Wertheim Salomonson, Ein neues Reizungsgesetz. Verslag der Koninkl. Acad. 1902 S. 385, 486, 610; Bd. 41 (deel II) S. 472, 539. Die Effektgrösse als Funktion der Reizgrösse. Pflüger's Arch. Bd. 100 S. 455. 
Die Richtigkeit dieses Gesetzes, das also die Effektgrösse als Funktion der Grösse des Reizes bezeichnet, ist geprüft worden an zablreichen Untersuchungen, bauptsächlich Tigerstedt's, Waller's und des einen der Verfasser, Untersuchungen, die vorgenommen wurden an Froschmuskeln, an Nerven und am N. opticus. Aus der Art und Weise, wie die Formel abgeleitet worden ist, geht die Beschränkung hervor, dass das Reizungsgesetz nur gültig sein kann für Reize von sehr kurzer Dauer, die, praktisch gesprochen, bereits vollständig vorüber waren, bevor der Effekt begann.

Auf Grund hauptsächlich mathematischer Überlegungen, und gestützt auf einige bei $W$ aller ${ }^{1}$ ) verzeichnete physiologische Tatsachen, musste angenommen werden, dass Reize, die auf ein Endorgan ausgeübt werden, bei ihrem Übergange auf höhere Neuronen in diesen einen Effekt hervorriefen, dessen Grösse durch dieselbe Formel ${ }^{2}$ ) bezeichnet wurde. Hieraus müsste folgen, dass einige Aussicht bestände, auch die psychisch wahrgenommene Intensität eines Reizes und die physische Grösse desselben durch unsere Formel ausdrücken zu können.

Hierfür spreehen einige Tatsachen. Zuerst die Tatsache, dass für einzelne sinnlichen Wahrnehmungen ohne Zweifel eine maximale Empfindung angenommen werden kann, über die hinaus keine intensivere Wahrnehmung möglich ist. Man denke an erster Stelle an die Empfindungen von warm und kalt, die mit ziemlich grosser Sicherheit ein gewisses Maximum nicht überschreiten können. Wohl gibt es in unserem Organismus Hilfsmittel, die uns in den Stand setzen, über dieses Maximum scheinbar hinauszugehen, z. B. unsere Appreziation bezüglich der Wärmestrahlung auf Endorgane, die verbältnismässig weit entfernt von dem Endorgan liegen, das wir zu reizen wünschen. Dann der Schmerz, den wir fühlen, wenn eine bestimmte Temperatur äberschritten wird usw.; doch diese sollen hier ausser Betracht bleiben.

Auch hinsichtlich unserer Gewichtsempfindung besteht vermutlich ein Maximum: dieses Maximum ist erreicht, sobald wir nicht mehr imstande sind, ein bestimmtes Gewicht zu heben. Auch hier haben wir abzusehen von allerlei Hilfsmitteln, die es uns ermöglichen,

1) Waller, Brain 1895 S. 209.

2) J. K. A. Wertheim Salomonson, Ein neues Reizungsgesetz. Verslag đer Konink1. Akad. 1902 S. 385, 610. Siehe auch Anhang I. 
unter günstigen Umständen ausser dem Schwersten noch etwas Schwereres zu unterscheiden.

Ein zweites Argument gewinnen wir aus der Tatsache, dass eine Reihe von Untersuchungen, die angestellt wurden, um die Richtigkeit des Weber'schen Gesetzes zu erweisen, in der Regel ein Minimum liefert.

Wie bekannt, hatte Weber bemerkt, dass zwei Sinnenreize, zwischen denen noch ein Unterschied wahrgenommen werden konnte, in konstantem Verhältnis zueinander standen; oder auch, dass der Unterschied zwischen diesen beiden Reizen stets einen g]eich grossen Teil der physischen Grösse eines der Reize bildete, gleichgültig, wie gross dieser auch war. War man z. B. imstande, mittels des Gefühls $30 \mathrm{~g}$ von $31 \mathrm{~g}$ zu unterscheiden, so konnte man auch gerade noch 300 und $310 \mathrm{~g}$ oder 3000 und $3100 \mathrm{~g}$ unterscheiden.

Fechner nahm an, dass ein gerade noch merkbarer Unterschied zwischen zwei Wabrnehmungen eine konstante psychische Grösse darstelle und durch eine konstante mathematische Grösse wiedergegreben werden könne. Hieraus konnte er das bekannte psychophysische Gesetz ableiten, welches lautet: Wenn ein Reiz nach einer geometrischen Reihe zunimmt, dann nimmt die Wahrnehmung $\mathrm{zu}$ in arithmetischer Reihe.

Das Weber'sche Gesetz ist immer wieder aufs neue untersucht worden. Man hat Tausende von Untersuchungsreihen ausgeführt, um die Richtigkeit desselben zu beweisen, und das Resultat ist gewesen, dass grosse Meinungsverschiedenheit über seine Richtigkeit herrscht. Ein Untersucher, der von einem Gesetze allein verlangt, dass es so ungefähr stimme, ist auch zufriedengestellt durch die Resultate der zahlreichen Untersuchungen über das Gesetz Weber's. Wer jedoch mehr verlangt, stösst sofort auf zwei Schwierigkeiten:

1. auf die sogenannte untere Abweichung von dem Gesetze We ber's;

2. auf die sogenannte obere Abweichung von dem Weber'schen Gesetze.

Wenn damit alle Abweichungen aufgezählt wären, dann könnte man sich gut mit dem Weber'schen Gesetze vereinigen und sagen, es sei eine gute Interpolationsformel für ein bestimmtes, sogar ziemlich weites Gebiet (Langelaan) ${ }^{1}$ ).

1) Langelaan, Versl. d. Koninkl. Akad. v. Wetenschappen 1902 S. 855. 
Es ist jedoch noch eine andere Abweichung vorhanden, und zwar folgende: Jede genügend umfassende Reihe von Untersuchungen, die mit der nötigen Genauigkeit vorgenommen werden, zeigt ein Minimum. Und dies gilt für jedes Sinneswerkzeug. Am sprechendsten sind vielleicht Fechner's eigene Zahlen, was den Gewichtssinn anbelangt. Gleich überzeugend sind die Zahlen, die Aubert, Helmholtz, König und so viele andere ermittelten.

Auch aus dem schon oben genannten Reizungsgesetz (1) lässt sich leicht ein Ausdruck ableiten ${ }^{1}$ ) für die Grösse der relativen Unterschiedsschwelle des Reizes, wenn man ausgeht von der Hypothese Fechner's, dass ein gerade noch merklicher Unterschied zwischen zwei Reizen eine konstante Effektvermehrung repräsentiere. Nennen wir die Untersehiedsschwelle $\varrho$, dann lautet der aus dem Reizungsgesetz abgeleitete Ausdruck:

$$
\varrho=\hbar \frac{E^{\beta}}{R}
$$

In diesem Ausdrucke bezeichnen $k$ und $\beta$ konstante Grössen, während $R$ den Reiz vorstellt.

Wenn wir auf Millimeterpapier eine Linie ziehen, die graphisch die beiden genannten Formeln wiedergibt, dann wird Formel (2) durch eine gebogene Linie dargestellt, die die konvexe Seite der horizontalen Achse zukehrt und also ein Minimum zeigt.

Das Gesetz Weber's ist durch eine horizontale Linie darzustellen.

Die Tatsache, dass in fast allen Untersuchungsserien Fechner's, Helmholtz', König's, Aubert's usw. ein Minimum vorhanden ist, dürfen wir auch nie aus den Augen verlieren, wenn wir die Möglichkeit erwägen, ob das Reizungsgesetz (1) auch vielleicht irgendwelche Bedeutung habe für unsere Sinneswahrnehmungen.

Schon oben wurde darauf hingewiesen, dass das Reizungsgesetz ganz gewiss nur angewendet werden dürfe bei äusserst kurz wäbrenden Reizen. In der Literatur fanden wir keine für unseren Zweck geeigneten Versuchsreihen verzeichnet. Wir haben darum getrachtet, diese Lücke selbst zu ergänzen, und nahmen zu diesem Zwecke eine grosse Anzahl Versuchsserien vor, ursprünglich, wie gesagt, einzig und allein zu diesem $\mathrm{Z}$ wecke. Sehr bald zeigte es sich jedoch, dass wir eigentümliche Resultate erhielten, die sich weder mit dem

1) Siehe Anhang III. 
Reizungsgesetz noch mit dem Gesetze Weber-Fechner's in Einklang bringen liessen.

Wir haben hierauf unsere Versuchsbedingungen erst etwas verändert und die Versuche später auf eine mehr systematische Weise ausgeführt. Die Resultate, die wir schliesslich dabei erhielten, und die wir in nachstehendem Bericht wiedergeben, werden hoffentlich etwas Licht verbreiten über die physischen Bedingungen des We ber Fechner'schen Gesetzes.

\section{Reizung vieler Netzhautelemente.}

\section{A. Experimenteller Teil.}

1. Einrichtung der Versuche.

Nachdem sich unser Auge vollkommen an die Beleuchtungsintensität eines Raumes gewöhnt hatte, in dem die Beleuchtung einen gewissen konstanten Wert besass - es geschah dies durch einen Aufenthalt von längerer Iauer: in der Regel von mindestens 20 Minuten, in diesem Raume - , begannen wir mit unseren Versuchen. Diese bestanden darin, dass dem wahrnehmenden Auge während eines kurzen Augenblickes von bekannter, vorläufig unveränderlichen Dauer ein rundes, beleuchtetes Feld gezeigt wurde, dessen eine Hälfte stärker beleuchtet war als die andere. Wir wiederholten diesen Versuch mit kleinen Abänderungen in der Beleuchtungsintensität der einen Hälfte, bis der kleinste Unterschied in der Lichtstärke der beiden Hälften, der gerade noch mit Sicherheit als solcher erkannt wurde, erreicht worden war. Das wurde viele Male wiederbolt, jedesmal bei einer anderen absoluten Lichtstärke, so dass schliesslich die ganze Reihe der uns zu Gebote stehenden Lichtintensitäten durchlaufen war.

Mit ängstlicher Sorgfalt haben wir nach einem vollkommen konstanten Adaptationszustand während jeder Beobachtungsreihe getrachtet.

\section{Instrumentation.}

Das gebrauchte Instrumentarium war nahezu vollständig nach der von Müller-L yer ${ }^{1}$ ) angegebenen Methode zusammengestellt. Auf einem Tische wurde ein langer in- und auswendig schwarzer,

1) Müıler-Lyer, Du Bois-Reymond's Arch. 1887 S. 402. 
lichtdicht abgeschlossener Kasten aufgestellt. Eine Zwischenwand schied diesen in eine längere hintere und eine kürzere vordere Abteilung; in der Zwischenwand befand sich eine mit Seidenpapier überklebte Öffnung. Der Durchmesser dieser Öffnung betrug bei einem Teile unserer Versuchsreihen $10 \mathrm{~cm}$, bei einem anderen Teile $1 \mathrm{~cm}$; bei einem dritten Teile wurde der Durchmesser noch 2,5 mal verkleinert mit Hilfe eines umgekehrten Galiläi'schen Fernrohres. Die Vorderseite des Seidenpapiers wurde beleuchtet von zwei mit Reflektoren versehenen Glühlampen von 16 Kerzen Leuchtstärke; sie waren, dem wahrnehmenden Auge unsichtbar, aufgestellt in einer ständigen Entfernung von $32 \mathrm{~cm}$ vom Schirm. Überdies wurde die eine Hälfte des runden Seidenpapiers noch besonders beleuchtet mittels einer anderen Glühlampe von 16 Kerzen Leuchtstärke, die sich in der hinteren Abteilung des Kastens hefand; die andere Hälfte bedeckte an der Rückseite ein schwarzer Schirm, so dass sie auf diese Weise von ebengenannter Lichtquelle nicht beschienen werden konnte. Die eine Hälfte des weissen Papierfeldes konnte also nur auffallendes Licht nach der Vorderseite des Kastens werfen, die andere Hälfte dagegen vermochte ausser diesem zurückgeworfenen Licht auch Licht durchzulassen, das von der Lampe in der hinteren Abteilung herrührte.

So entstand ein Unterschied in der Lichtstärke, und dieser Unterschied konnte dadurch, dass die Lampe im hinteren Teile des Kastens vor- und rückwärts geschoben wurde, vergrössert oder verkleinert werden.

Um das beleuchtete Feld wahrnehmen zu können, befand sich in der Vorderwand des Kastens eine kleine Öffnung und daneben. eine Stütze für die Nase. Die Öffnung bedeckte ein Fallschirm; liess der Beobachter denselben fallen, so bewegte sich eine Öffnung im Schirm vor der Öffnung der Vorderwand vorüber, und das beleuchtete Feld wurde einen Augenblick sichtbar. Das beleuchtete Feld war in seiner Ebene drehbar, so dass die Grenzlinie zwischen beiden Hälften in den verschiedensten Richtungen dem Beobachter gezejgt werden konnte.

Über und unter der Öffnung waren an der Vorwand zwei Leisten mit Nuten befestigt; in dieselben konnten Rauchglasplatten aus- und eingeschoben werden. Mit Hilfe einer Anzahl dieser Platten konnte die absolute Lichtstärke reguliert werden.

Ein Fixationszeichen, das wir seitwärts aufgestellt hatten, und 
welches dem nicht beobachtenden Auge in einem Spiegelchen unter einem Winkel von $45^{0}$ mit der Gesichtslinie sichtbar wurde, brachte uns mehr Last als Bequemlichkeit und wurde deshalb ausser Gebrauch gesetzt.

\section{Gang der Beobachtungen.}

Der Beobachter brachte nach erlangter Adaptation das Auge vor die mittels des Fallschirmes geschlossene Öffnung in der Vorderwand des Kastens, während der andere dem runden Felde, das stets $40 \mathrm{~cm}$ vom Auge entfernt war, und auch der binteren Lampe eine willkürlich gewählte Stellung gab.

Hierauf liess der Beobachter dann selbst den Schirm fallen und gab an, welche Hälfte am stärksten oder welche am schwächsten beleuchtet war, und wie die Grenzlinie zwischen beiden verlief. War die Angabe richtig, so wurde dieselbe Beobachtung wiederholt, nachdem der Assistent die hintere Lampe etwas weiter zurückgeschoben und dem beleuchteten Kreise durch Drehen eine andere willkürlich gewählte Stellung gegeben hatte.

So ging es weiter, bis ein Punkt erreicht worden war, bei dem der Unterschied in der Beleuchtung der beiden Hälften gar nicht mehr oder nicht mehr gut wahrgenommen werden konnte, und ein Punkt, bei dem dies gerade noch möglich war.

Die ersten Bestimmungen führten wir aus mit ein paar sehr dunklen Rauchgläsern im Schieber; darauf wurde die Lichtstärke in grossen Sprüngen verstärkt, so dass wir mit einer Anzahl von fünf Bestimmungen ein Bild gewannen, wie sich der Gang der Arbeit auf dem ganzen Gebiete, von der schwächsten Beleuchtung bis zur stärksten, gestalten werde.

Nachdem dies genugsam festgestellt worden war, wurden die Bestimmungen für den Teil des Gebietes wiederholt, der sich als der wichtigste herausgestellt hatte, während wir dabei jetzt eine viel langsamere Steigerung der Lichtstärke eintreten liessen.

Unsere Untersuchung fand statt bei vier Adaptationszuständen:

1. während das Auge des Beobachters sich der absoluten Finsternis (in der Dunkelkammer) adaptiert hatte;

2. bei Adaptation an eine Beleuchtung von 5,5 Meterkerzen, gemessen mit Martens' ${ }^{\prime}$ ) Photometer (in einem von ein paar Glüh-

1) Martens, Über ein neues Polarisationsphotometer. Physik. Zeitschr. Bd. 1 S. $299-303$. 
lampen erleuchteten, im übrigen vollständig gegen das Licht abgeschlossenen Zimmer);

3. bei einer solchen an eine Beleuchtung von etwa 50 Meterkerzen (in einem Zimmer mit dem reflektierten Lichte zweier Bogenlampen zeigte das Photometer 37 M. $K$. auf den dunklen Teilen der Wände an, 43 M.-K. auf den helleren Teilen in Manneshöhe, 60 M.-K. höher an den Wänden und 87 M.-K. auf einem weissen Schirm, der horizontal auf dem Tische lag);

4. bei Adaptation an eine Beleuchtung von reichlich 350 M.-K. (in einem Zimmer mit direkt einfallendem Sonnenlicht zur Zeit der grössten Helligkeit an sonnigen Frühlingstagen).

Die Beobachtungszeit blieb bei all diesen Versuchen dieselbe. Wir haben jedoch noch einige Reiben in unsere Untersuchung hereingezogen, bei denen die Zeit länger war; zu diesem Zwecke liessen wir den Fallschirm von einer geringeren Höhe fallen. Auch führten wir einige Reihen mit unbestimmt langer Beobachtungszeit aus, indem wir den Fallschirm einfach wegnahmen.

\section{Bericht über die gew onnenen Resultate.}

Unsere Untersuchung, die sich ungefähr über die Zeit eines Jahres erstreckte, bot, zumal im Anfang, die gewöhnlichen Schwierigkeiten, die mit einer derartigen Arbeit verbunden sind. Diese bestanden zunächst in der Ungeübtheit der untersuchenden Personen, ferner in der bald eintretenden Ermüdung.

Infolge dieser beiden Umstände war es darum nicht möglich, in jeder Sitzung mehr als zwei Reihen zu bestimmen, eine Reihe, bei der einer der Verfasser Untersucher war und der andere untersucht wurde, und die andere Reihe mit vertauschten Rollen.

In den ersten Sitzungen konnten deshalb nur einzelne Punkte der Kurve bestimmt werden, höchstens 6 oder 7 , wobei für jeden Punkt eine Anzahl von 10-15 einzelnen Reizen sich nötig machten.

Nach einigen Sitzungen zeigte es sich bald, dass infolge der gewonnenen Fertigkeit die Unterscheidungsgrenze fortwährend sank, bis schliesslich ziemlich konstante Werte erreicht waren.

Unter die unten folgenden Zahlen sind die Übungsserien nicht aufgenommen worden. Ebensowenig haben wir ferner alle Serien aufgenommen, sondern beschränken uns auf die Mitteilung der Resultate, die dadurch gewonnen wurden, dass wir für jeden Punkt 
einer Gruppe von Reihen ein und derselben Art den Durchschnitt suchten.

Damit geben wir das wieder, was nach unserer Meinung der Wahrheit möglichst nahekommt. Es ist möglich, dass etwas grössere Genauigkeit erreichbar ist, - doch meinen wir, dass die Schwierigkeit und die Mühe, die das Streben nach noch grösserer Genauigkeit verursachen würde, nicht durch eine entsprechende Steigerung in den Werten der Resultate belohnt werden könne.

Da für jede Reihe eine Anzahl von 80-100 Wahrnehmungen nötig waren und wir im ganzen ungefähr 50-60 Reihen bestimmt haben, so ist die Anzahl individueller Versuche annähernd an die $5-6000$ gewesen.

Erst nachdem wir alle unsere Versuche beendet hatten, stellten wir die zu einer genauen Berechnung der Resultate nötigen Daten zusammen.

In verkurzter Form und besser, als jede Beschreibung es vermag, gibt die nacbstehende Tabelle I die Resultate unserer Untersuchung wieder.

Man findet in der ersten Kolumne das Merkzeichen der Rauchglasplatte oder -platten. Die folgenden Vertikalreihen geben an, bei welchen Entfernungen der hinteren Lampe die Unterschiedsschwelle, erreicht worden war, und zwar für jeden der beiden Wahrnehmer besonders und in dem direkt wahrgenommenen Bilde. Näheres über das Nachbild wird sogleich folgen.

Hier muss bemerkt werden, dass die in Centimetern angegebenen Entfernungen gemessen sind nicht vom beleuchteten Felde, sondern von einem willkürlichen Nullpunkte aus, da man bei einer Lichtquelle von einiger Grösse die Lichtstärke nicht in umgekehrtem Verhältnis zu dem Quadrat der Entfernung stehend ansehen darf, wenn weuigstens diese Entfernung im Hinblick auf die beleuchtete Oberfläche klein ist; experimentell muss bestimmt werden, welche Lichtstärken den gefundenen Entfernungen entsprechen. Auch meine man nicht, dass die Messungen über ein Centimeter genau seien; man würde sich damit eine Genauigkeit und Schärfe der Apparate vorspiegeln, die nicht möglich ist; die Dezimalen in der Tabelle sind allein dadurch entstanden, dass die Zahlen aus mehreren Reihen als Durchschnittszahlen gewonnen wurden; und weil zuweilen eine Korrektur der Zahlen wegen kleiner Veränderungen am Apparat vorgenommeu werden musste. 
Die Angaben in der Tabelle sind so angeordnet, dass man zunächst alle Wahrnehmungen verzeichnet findet, die bei sehr kurzer Wahrnehmungszeit gemacht wurden, dann diejenigen an den Objekten von $10 \mathrm{~cm}, 1 \mathrm{~cm}$ und $4 \mathrm{~mm}$ Durchmesser bei verschiedenen Adaptationszuständen. Es folgen dann einige Reihen mit längerer Beobachtungszeit und schliesslich solche von unbestimmt langer Dauer, von denen vor allem die beiden ersteren mit sehr langer Wahrnehmungszeit ausgeführt worden sind. Zur Erleichterung von Hinweisen ist jede Vertikalreihe mit einem Buchstaben bezeichnet, den man in anderen Tabellen und Figuren wiederfinden wird, die auf dieselben Wahrnehmungsreihen Bezug haben.

(Siehe Tabelle I S. 399.)

\section{B. Rechuerischer Teil.}

\section{Die Wahrnehmungszeit.}

Um zu berechnen, wie lange das beleuchtete Feld jedesmal sichtbar gewesen war, wurde auf dem Fallschirm ein berusster Papierstreifen befestigt; ein Deprez-Marey'sches Signal, welches 100 Schwingungen in der Sekunde machte, wurde dagegengesetzt. Aus der Linie, die die Feder unter dem Fallen des Schirmes auf dem berussten Streifen beschrieb, konnte abgelesen werden, dass bei Anwendung der grössten Fallhöhe die Dauer der Sichtbarkeit 0,0153" betrug, wenn man annimmt, dass die Pupille im absoluten Dunkel $8 \mathrm{~mm}$ weit ist ${ }^{1}$ ).

Berechnet man die Wahrnehmungszeit aus den Formein für die Fallhöhe, dann findet man $0,01345^{\prime \prime}$; sie ist kleiner als die vorige, da hier die Reibung nicht in Rechnung gezogen ist; der auf experimentellem Wege gefundene Wert ist aus demselben Grunde genauer. Daraus ist ersichtlich, dass Veränderungen in der Weite der Pupille bei all diesen Experimenten ausser Betracht bleiben können; denn diese Kontraktion tritt erst $0,33 "$ (Vintschgau) oder selbst 0,49" (A rlt-Donders) nach Beginn des Lichtreizes ein.

Dasselbe gilt von der geringen Fallhöhe, die wir angewendet haben. Dauerte doch die Sichtbarkeit nur 0,040".

1) Die Pupille des einen der Verfasser, die im Adaptationszustand an dunkel photographiert wurde, ehe sie zu reagieren vermochte, ergab einen Durchmesser von $8,35 \mathrm{~mm}$. 
Tabelle I.

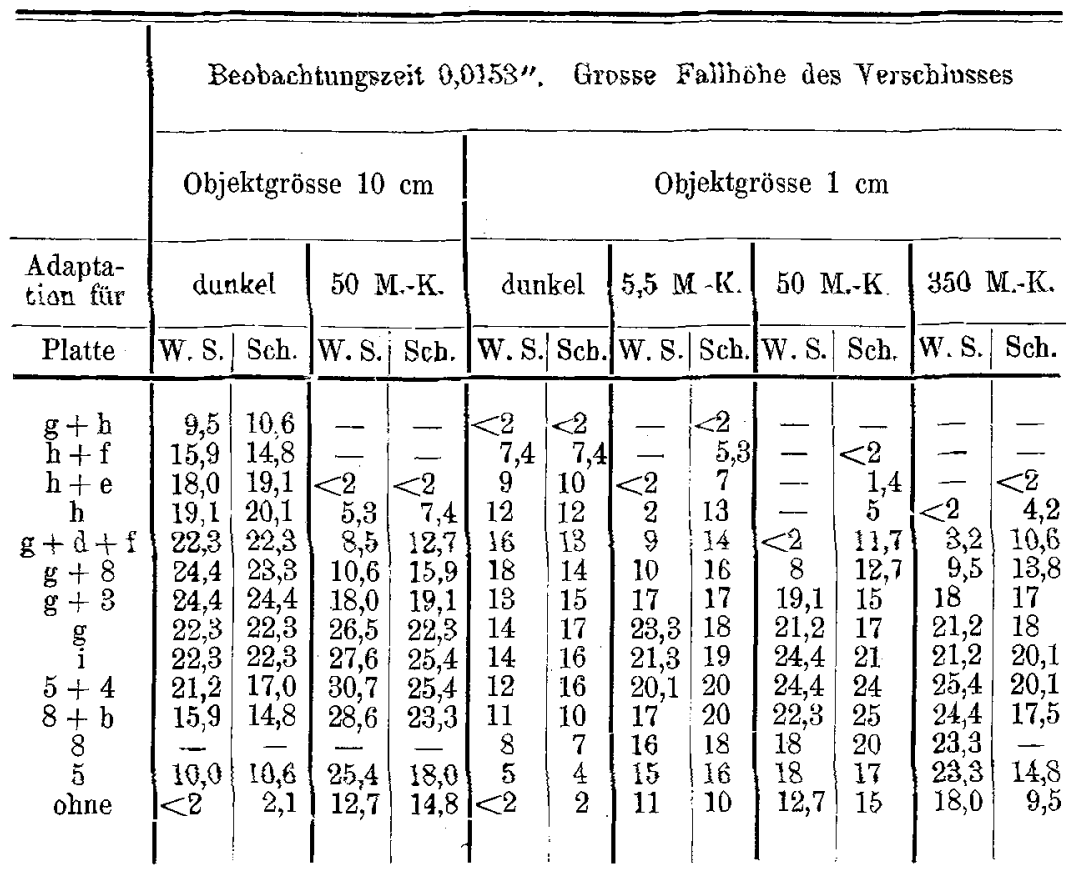

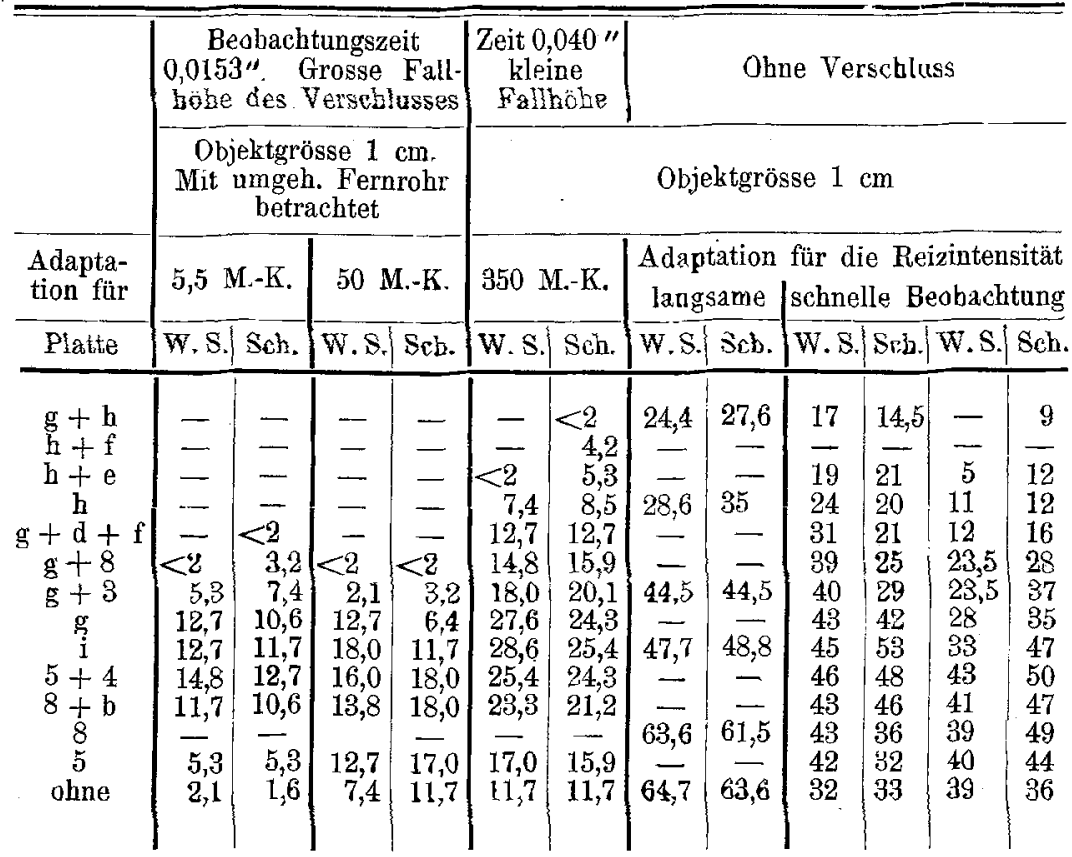


Wir kommen übrigens sogleich noch auf den Einfluss der Pupillenweite zurück.

2. Die Exstinktionskoeffizienten der Rauchgläser.

Diese haben wir aus einer sehr grossen Anzahl Bestimmungen berechnet, die wir teils mit dem Photometer von LummerBrodhun, teils mit dem neuen Photometer von Martens gewannen; letzteres hat uns besonders befriedigt wegen der bequemen und genauen Weise, in der es eingestellt werden kann.

Bei dieser Gelegenheit sei ein Wort des Dankes Herrn Dr. J. Boeke dargebracht, der so freundlich war, eine Anzahl dieser Bestimmungen für uns auszuführen.

Die rechnerisch gewonnenen Durchschnitte werden unten angegeben. Die Anzahl Rauchgläser betrug 17. Die Anzahl der Bestimmungen, aus denen der Durchschnitt gewonnen wurde, betrug $50-100$ per Glas.

$\begin{array}{cccc}\text { Platte } & \begin{array}{c}\text { Exstinktions- } \\ \text { vermögen }\end{array} & \text { Platte } & \begin{array}{c}\text { Exstinktions- } \\ \text { vermögen }\end{array} \\ 1 & 1,36 & \mathrm{a} & 1,30 \\ 2 & 2,37 & \mathrm{~b} & 2,05 \\ 3 & 3,08 & \mathrm{c} & 2,30 \\ 4 & 4,37 & \mathrm{~d} & 3,48 \\ 5 & 6,05 & \mathrm{e} & 3,81 \\ 6 & 4,19 & \mathrm{f} & 6,04 \\ 7 & 5,28 & \mathrm{~g} & 122,00 \\ 8 & 8,00 & \mathrm{~h} & 5820,00 \\ & & \text { i } & 69,00\end{array}$

3. Beleuchtung des beobachteten Feldes durch die vorderen Lampen.

Diese Lichtstärke wurde gemessen mit dem Martens'schen Polarisationsphotometer. Zuvor bestimmten wir die drei Konstanten dieses Instrumentes mit Hilfe einer geeichten Hefnerlampe, die uns von Herrn Professor Saltet liebenswürdig zur Verfügung gestellt wurde.

Wir fanden

$$
\begin{aligned}
& \mathrm{C}_{1}=27,523 \text { (W. S) und } 26,451 \text { (Sch.), } \\
& \mathrm{C}_{2}=\mathrm{C}_{1} \text { und } \\
& \mathrm{C}_{3}=0,0385 \text { (für beide Beobachter). }
\end{aligned}
$$

Nachdem dies geschehen war, wurde das Photometer auf die Vorderseite des runden Feldes gerichtet, während dieses einzig und 
allein von den beiden Lampen in der vorderen Abteilung des Apparates beleuchtet wurde. Dabei fanden wir eine Lichtstärke von $0,01175 \mathrm{H} . \mathrm{K}$. auf $1 \mathrm{qcm}$; diesen Wert nehmen wir vorläufig als Einheit an.

4. Beleuchtung des beobachteten Feldes durch die hintere Lampe.

Das Photometer hat dieselbe Stellung wie vorher. Die hintere Lampe wird so aufgestellt, dass die im Photometer wahrgenommene Beleuchtung unveränderlich bleibt, wenn man das Seidenpapier abwechselnd mittelst der vorderen Lampen beleuchtet oder mittelst der hinteren Lampe allein. Dies war der Fall, als die hintere Lampe auf Teilstrich 7 stand; bei diesem Stande wurde also offenbar das runde Feld von der hinteren Lampe beleuchtet mit einer Menge $I$ durchfallenden Lichtes.

Ferner wurde die Lichtstärke des runden Feldes bei sechs anderen Stellungen der hinteren Lampe bestimmt. Diese sechs Werte, die auf sechs an der Skala abgelesene Werte Bezug haben, werden benutzt, um eine graphische Darstellung zu konstruieren, die zeigte, wie die Lichtstärke sich beim Verschieben der Lampe von Teilstrich zu Teilstrich änderte. Die Konstruktion wurde durch Interpolation kontrolliert. Der für Teilstrich 7 bestimmte Wert, der der Beleuchtung mittelst der vorderen Lampen gleich befunden wurde, also $=R$, wurde als Einheit angenommen; die Zahlen geben dann direkt an, wie gross der Wert $\frac{d R}{R}$ auf jedem Teilstrich ist.

Die Lichtstärke wird berechnet nach der Formel $J=C_{3} t g^{2} \alpha$, in welcher $C_{3}$ die obenerwähnte Konstante und $\alpha$ das Komplement eines Winkels ist, der am Photometer abgelesen wurde. $I$ steht also in einem bestimmten Verhältnis zum Quadrat der genannten Tangente.

Wenn es also einen Nullpunkt gibt, von dem aus gerechnet die Lichtstärke in umgekehrtem Verhältnis zu den Quadraten der Entfernungen steht, dann muss diese Entfernung wieder in umgekehrtem Verbältnisse zur Tangente des genannten Komplements stehen oder in geradem Verhältnis zur Tangente des abgelesenen Winkels.

Trägt man also einige Entfernungen auf einer Abscissenachse $a b$ und konstruiert die Tangenten der abgelesenen Winkel als dazu gehörende Ordinaten, dann müssen erstens die Spitzen dieser Ordi. 
naten in einer geraden Linie liegen und muss zweitens diese gerade Linie die Abscissenachse in dem unbekannten Nullpunkte schneiden.

Es zeigte sich, dass unsere sechs Bestimmungen auf unerwartet genaue Weise der ersten Forderung genügten, was uns die Gewissheit gibt, dass wir ganz brauchbare Werte bestimmt haben. Der Nullpunkt lag, wie sich herausstellte, $9,8 \mathrm{~cm}$ vor Teilstrich 0 . Zählt man also zu allen abgelesenen Grössen $9,8 \mathrm{~cm}$ hinzu, so kann man das Verhältnis der Lichtstärken aus den umgekehrten Quadraten dieser korrigierten Entfernungen berechnen.

\section{Berechnung der Resultate.}

Wir haben jetzt alle Daten beisammen, um den Lauf des Wertes $\frac{d R}{R}$ von der schwächsten bis zur stärksten Beleuchtung berechnen zu können; eine Übersicht hierüber geben wir in der folgenden Tabelle II auf S. 15 u. 16.

Es finden sich in dieser Tabelle II dieselben Beobachtungen verzeichnet wie in Tabelle I. Die Bezeichnung der Glasplatten ist jedoch ersetzt durch ihre Exstinktionskoeffizienten. . Darauf folgt die absolute Liehtstärke $R$.

Würden wir nun noch, wie dies soeben geschah, die grösste Lichtstärke 1 nennen, dann erhielten wir für alle anderen Werte Brüche, was Sehwierigkeiten verursacht; wir nennen darum nun die schwächste Beleuchtung 1: die stärkste wird dann 650260, und die dazwischenliegenden Werte findet man, wenn man diese Zahl durch die Exstinktionskoeffizienten teilt.

Wir lassen alsdann noch die Logarithmen der Lichtstärken folgen, weil sie sogleich als Abscissen für eine graphische Darstellung dienen werden. In den übrigen Kolumnen findet man, übereinstimmend mit den Vertikalreihen der Tabelle I, die Werte für $\frac{d R}{R}$.

Während eine Tabelle dazu nötig ist, die jedesmal erhaltenen Werte der relativen Unterschiedsschwelle zu spezifizieren, wird eine graphische Darstellung bessere Dienste tun, um eine Übersicht über das Ganze zu geben.

Die auf der Achse der Abscissen abgetragenen Lichtstärken (siehe die Figuren) sind logarithmisch eingeteilt, da das Gebiet ihrer wirklichen Werte nicht auf dem Papier unterzubringen sein würde; die zugehörigen Schwellenwerte sind nicht logarithmisch, sondern in 
Psychooptische Untersuchnngen.

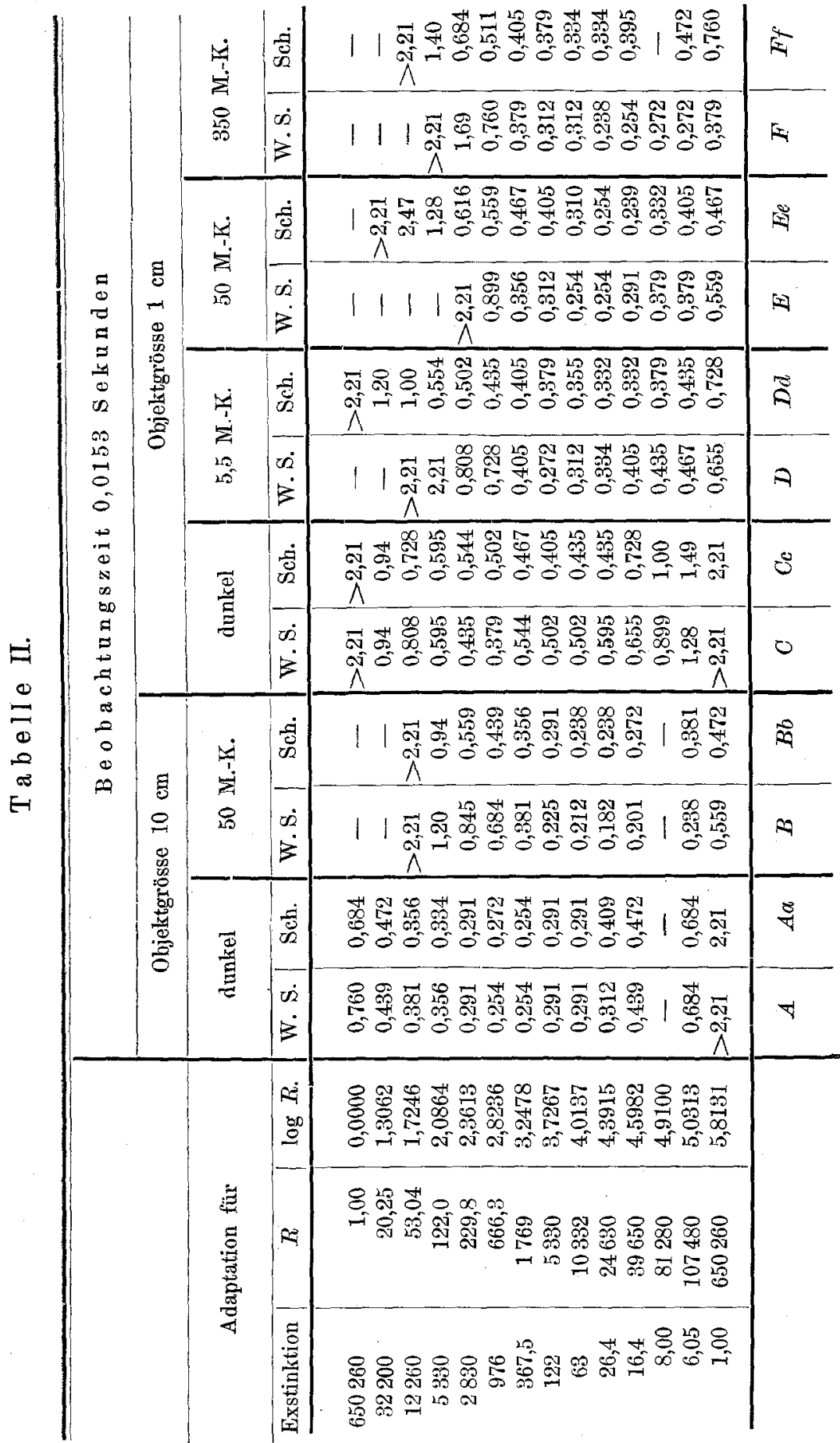




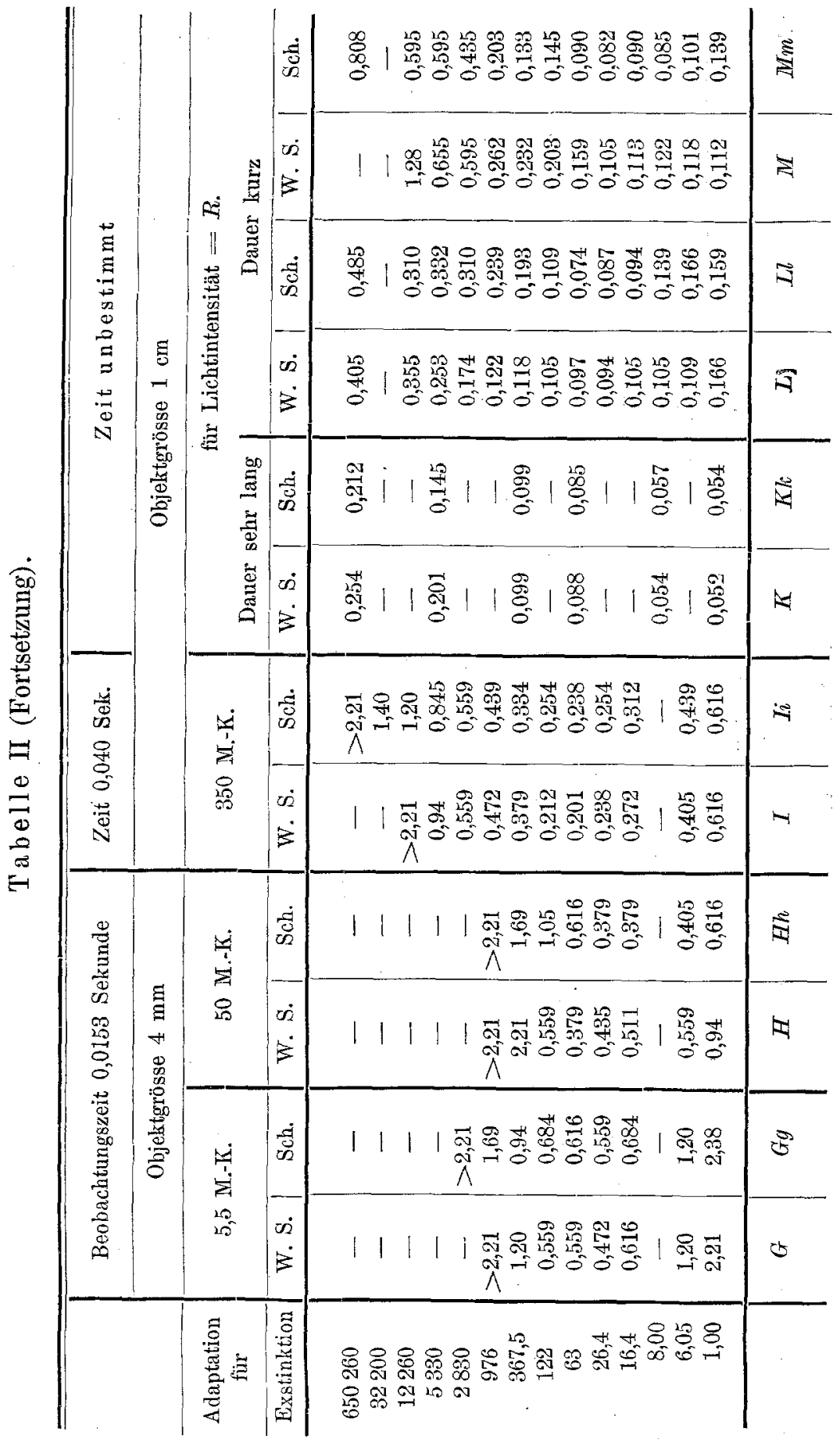


ihren wahren Grössen auf den Ordinaten abgetragen. Die Buchstaben, mit denen die Linien bezeichnet sind, geben deutlich genug an, auf welche Versuchsreihen sie Bezug haben.

Wir lassen nun an der Hand dieser Ergebnisse

folgen.

\section{c. Einige Betrachtangen}

Wer die Tabelle II durchsieht, dem wird es auffallen, dass unsere relative Unterschiedsschwelle so gross ist. Abgesehen von den Versuchen mit sehr langer Wahrnehmungszeit, bei denen wir im günstigsten Falle ${ }^{1 / 20}$ erhielten $(K)$, ist ihr Wert bei kurzer Beleuchtingsdauer nicht unter $1 / 5$ gesunken $(B)$. Dies hat jedoch nicht an dem untersuchenden Auge gelegen; denn mit einigen Masson' schen Scheiben erreichten wir schon bei dem ersten Versuche eine Empfindlichkeit von 1/180, sehr bald selbst von 1/2s2. Auch vermochten wir mit einer Vorrichtung, ähnlich ${ }^{1}$ ) der von König und Brodhun, 1/50 bis 1/70 zu erreichen, dieselben Werte, die auch diese angeben. Unsere höheren Werte haben also unzweifelhaft ihren Grund darin, dass das gestellte Problem sehr schwierig war.

Die graphischen Darstellungen sind konstruiert mit Hilfe der Zahlen, die wir aus unseren Wahrnebmungen gewonnen haben; man könnte vielleicht aus theoretischen Gründen die Meinung verteidigen, dass eine Korrektur erwünscht wäre: die Weite der Pupille ist, wie eben schon dargetan wurde, während jeder Versuchsserie unverändert geblieben; bei den verschiedenen Adaptationszuständen jedoch war sie jedesmal eine andere, und zwar wechselte sie von $8,35 \mathrm{~mm}$ in der Dunkelkammer bis $3 \mathrm{~mm}$ im hellen Sonnenlichte. Darum gibt die Menge Licht, die da s A uge traf, nicht die absolute Beleuchtung der Netzhaut wieder; diese lernen wir erst kennen, wenn wir die Tatsache in Rechnung ziehen, dass von der Lichtmenge nur ein Teil, im Verhältnis zur Weite der Pupille, ins Auge zugelassen

1) Hierzu benutzten wir wieder das schon mehrfach erwähnte Martens'sche Photometer, das ohne weiteres für Bestimmungen nach König-Brodhun's Anordnung benutzt werden kann, falls man an Stelle der Blende am Objektivende des Tubus eine solche mit je einer zentralen und einer peripheren, $5 \mathrm{~mm}$ weiten, direkt aneinander anliegenden öffnung einsetzt. Das kleine handliche Instrument sollte in keinem physiologischen Laboratorium fehlen. 
wird: die gefundenen Schwellenwerte gehören also tatsächlich erst zu den korrigierten absoluten Lichtstärken.

Aber die Schwierigkeit ist eine rein theoretische: in den graphischen Darstellungen sind, wie gesagt, nicht die Lichtstärken selbst, sondern die Logarithmen der Lichtstärken als Abscissen abgetragen.

Eine Teilung der Werte der Lichtstärken durch eine Zahl, z. B. durch 2, ändert nichts an der Form der Kurve, sondern verschiebt einfach diese Linie in toto ein Stück $=\log$. 2. Die Linien würden, ohne dass ihre Richtung sich änderte, etwas nach links geschoben werden.

Wir haben dies jedoch nicht getan, zunächst, um die Zeichnung nicht undeutlich zu macben, dann aber auch, weil wir wiederzugeben wünschten, was geschieht, wenn eine gewisse Menge Licht, die von einem Gegenstande von bestimmter Grösse ausgestrablt wird, in einer bestimmten Entfernung wahrgenommen wurde; die Menge Licht, die die Netzhaut traf, ist in unserem Falle erst von sekundärer Bedeutung.

Trachten wir nun, zu übersehen, was das Resultat unserer Untersuchung war, und halten wir dabei dieselbe Reihenfolge ein, die auch bei unserer Untersuchung beobachtet wurde.

Die ersten Versuche wurden vorgenommen bei Adaptation an die Dunkelheit und einer Objektgrösse von $10 \mathrm{~cm}$; die Entfernung: war $40 \mathrm{~cm}$, die Dauer des Lichtreizes betrug 0,0153 Sekunden; die Versuche wurden fortgesetzt, bis konstante Resultate erhalten worden waren. Die Kurven $A$ und $A a$ der Figuren 1 und 2 verkörpern die Ergebnisse dieser Serien.

Bei dieser Versuchsreihe zeigte sich alsbald eine Eigentümlichkeit. Während nämlich bei Anwendung der verschiedenen Lichtstärken, die geringer als 5330 waren, kein Augenblick gezweifelt wurde, dass die Empfindung des Unterschiedes in der Lichtintensität der beiden Hälften des beleuchteten Feldes in dem Augenblicke eintrat, da der Lichtreiz ausgeübt wurde, beobachtete man bei einer Intensität von 5330 , dass nach der Einwirkung des Lichtreizes zunächst ein deutliches Nachbild entstand; Unsicherheit jedoch herrschte nun dabei, ob der Unterschied in der Intensität der Hälften des Gesichtsfeldes unmittelbar wahrgenommen werde oder erst im $\mathrm{Nach}$ bilde.

Bei etwas höheren Lichtstärken verschwand diese Unsicherheit und konnte sehr leicht festgestellt werden, dass die direkte Licht- 
wahrnehmung blendete, so dass es nicht möglich war, irgend einen Unterschied zu erkennen, während etwa eine Sekunde später ein helles, scharfes Nachbild entstand, bei dem die Intensitätsunterschiede deutlich sichtbar waren.

Wir möchten hier gleich die Bemerkung machen, dass die Erscheinung nicht neu ist: sie wird schon von $\mathrm{Helmholtz}$ erwähnt (Physiol. Optik 2. Aufl. S. 503). Je höher die Lichtstärke, um so deutlicher trat sie zutage.

Von dem Punkte der Kurve ab, bei welchem das Nachbild zum Vorschein trat, führten wir zwei Reiben von Bestimmungen aus: wir bestimmten zunächst, welche Unterschiedsschwelle für $d a s$ $\mathrm{Nachbild}$ gilt, und wie gross die Unterschiedsschwelle beim direkten Bilde war. Es lag klar auf der Hand, dass wir mit zwei verschiedenen Prozessen zu tun hatten, von denen jeder absonderlich verfolgt werden musste. Eins war jedoch sicher, dass nämlich die Entstehung des Nachbildes, die bei den geringeren Lichtstärken nicht vorkam, sich als etwas Neues herausstellte. (In Figur 1 und 2 sind unter $A$ und $A a$ die beiden Stücke der Kurve wiedergegeben; der aufsteigende Ast gilt für die direkte Wahrnehmung, der untere punktierte Ast auf der rechten Seite für das Nachbild, so dass dieses besonders gewürdigt werden musste.)

Da bekanntlich bei einem Adaptationszustande des Auges an die Dunkelheit schon ein verhältnismässig schwacher Lichtreiz ein Nachbild gibt, was bei. Adaptation an Licht nicht der Fall ist, so meinten wir diese Schwierigkeit dadurch heben zu können, dass wir unsere Versuche bei einem Adaptationszustande an Licht wiederholten. So entstand eine zweite Gruppe von Versuchsreihen, ausgeführt in einem hellerleuchteten Zimmer, dessen mittlere Lichtstärke ungefähr 50 M.-K. betrug (Reihe $B$ und $B b$ ).

Es zeigte sich nun in der Tat, dass die eigentümliche Erscheinung, im Nachbild ein besseres Unterscheidungsvermögen zu haben als im direkten Bilde, bei dem zur Anwendung gebrachten Lichtreiz bis zu 60000 Einheiten verschwunden war; nur bei 650200 Einheiten war sie noch einigermassen fühlbar. Als später die Kurven $B$ und $B b$ (Fig. 1 und 2) gezeichnet waren, ergab sich, dass, praktisch ausgedrückt, die Kurven $A$ und $B$ resp. $A a$ und $B b$ kongruent und nur auf der Abscissenachse verschoben waren. Ferner stellte es sich heraus, dass die Kurven $A, A a, B$ und $B b$ einen typischen Verlauf zeigten: bei geringen Lichtstärken war die Ordi- 
408 J. K. A. Wertheim Salomonson und G. J. Schoute:

Fig 1

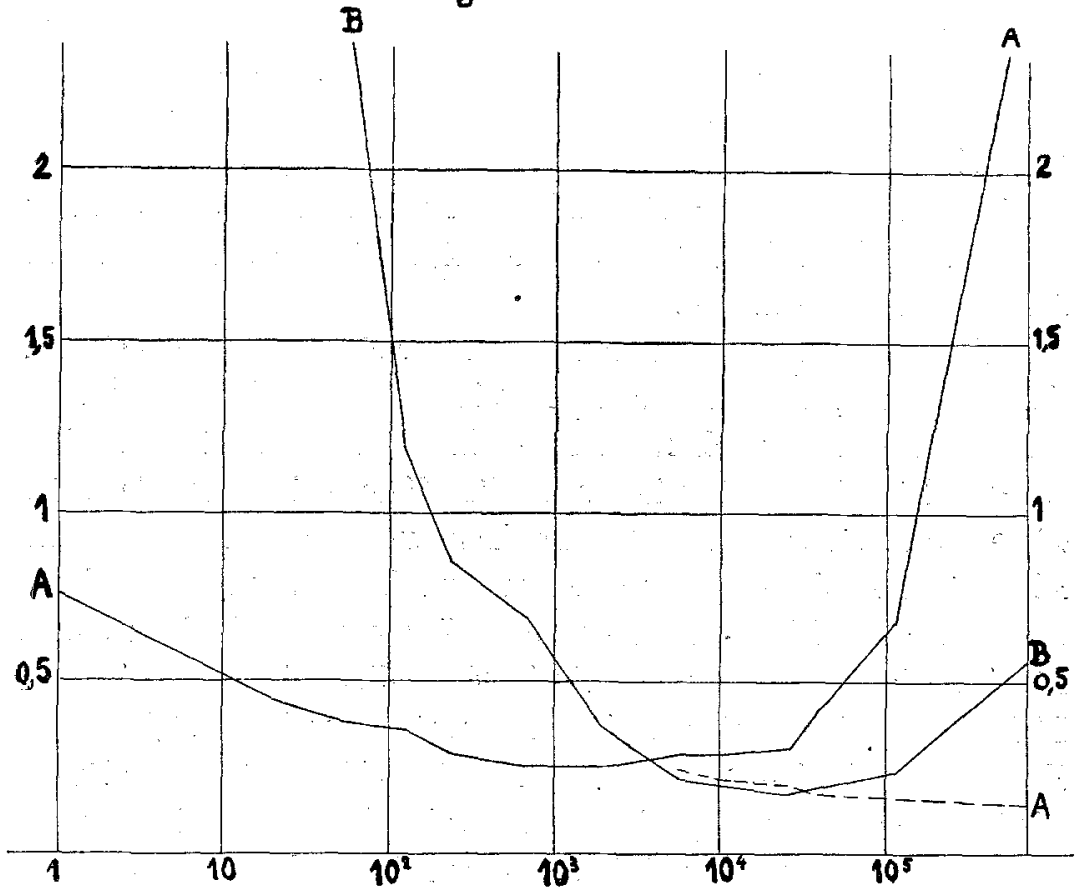

Fig 2

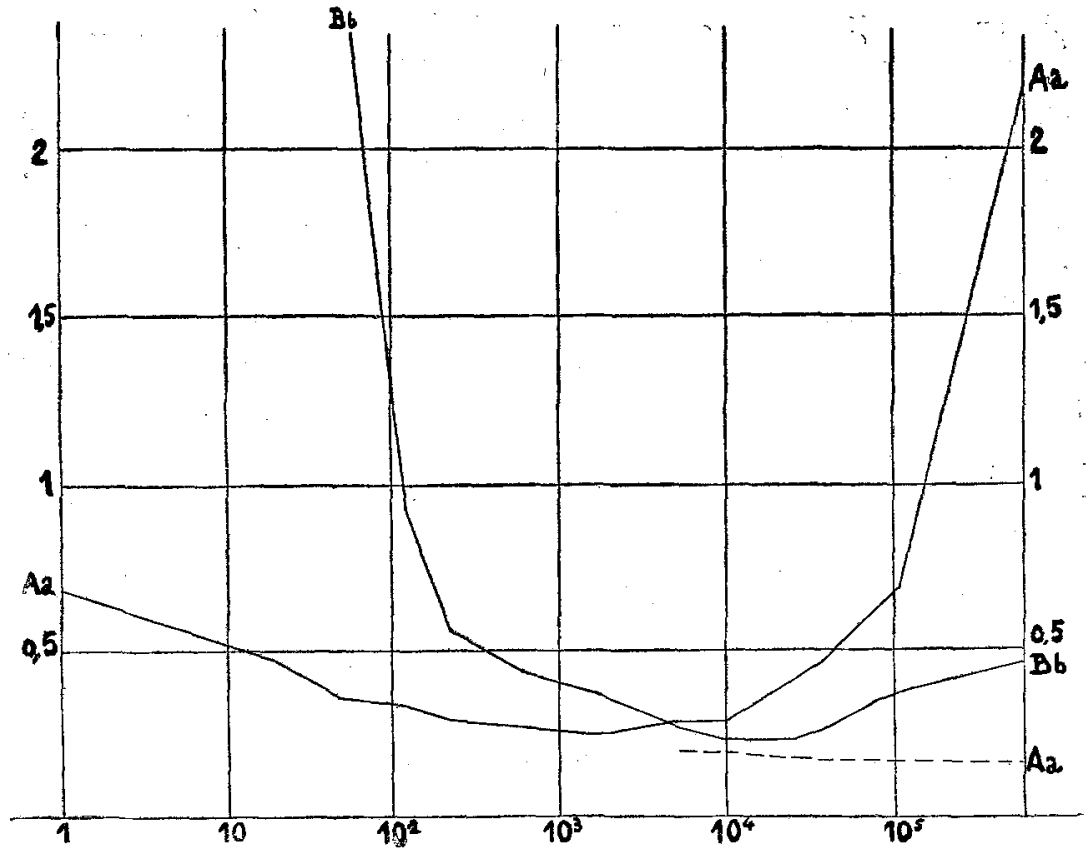


nate sehr hoch; bei Vermehrung der Lichtstärke trat ein allmähliches Sinken ein, bis ein Minimum erreicht war.

Von diesem Punkte ab begann die Kurve wieder zu steigen, zuerst langsam, bald aber rascher. Sie blieb immer konvex nach dem Koordinatensystem.

Da diese Erscheinung in jeder Versuchsreibe mit grösster Deutlichkeit zutage trat, so dürfen wir dies als das erste Ergebnis unserer Versuche betrachten. Zugleich gibt dieses Resultat die absolute Sicherheit, dass für Reize von kurzer Dauer das WeberFechner'sche Gesetz nicht mehr gilt und selbst nicht einmal für ein kleines Gebiet als Interpolationsformel angewendet werden darf.

Eine einfache Prüfung der erbaltenen Zahlen an der aus dem Reizungsgesetz (1) abgeleiteten Formel (2) lehrt jedoch ohne weiteres, dass diese Formel ebensowenig anwendbar ist.

Da es uns jedoch wahrscheinlich vorkam, dass die Form der Kurve, die wir erhalten hatten, von verschiedenen Umständen abbängig sei, u. a. von der Dauer des Reizes und der Anzahl Netzhautelemente, die jedesmal gereizt wurden, haben wir danach getrachtet, eine gewisse Einsicht zu erlangen in die Veränderungen, die in der Kurve entstehen, wenn wir diese beiden Faktoren veränderten.

Wir begannen also eine neue Versuchsreihe, bei welcher die beleuchtete Scheibe bis auf $1 \mathrm{~cm}$ verkleinert wurde, während die Entfernung zwischen Auge und Scheibe unverändert blieb. Nachdem auch hier einige Übungsserien vorausgegangen waren, erhielten wir endlich wieder ziemlici konstante Werte (Reihe $D, D d, E, E e$, $F, F f$, die allein darin sich voneinander unterschieden, dass bei $C$ und $C c$ unsere Augen sich an die Dunkelheit adaptiert hatten, während bei den folgenden Reihen eine Adaptation an Lichtstärken von 5,5-50 und 350 M.-K. bestand.

Bei diesen. Versuchsreihen zeigte es sich zunächst, dass die Schwierigkeit, mit der wir bei unseren ersten Versuchen zu kämpfen gehabt hatten, nämlich die Ungewissheit, ob wir im direkten Bilde oder im Nachbilde wahrnähmen, fast gänzlich verschwunden war. Nur bei den stärksten Reizen der Serien $C$ und $O c$ machte sich die Erscheinung in ziemlich geringem Masse bemerklich. Wir haben uns darum bei diesen Versuchen an die Bestimmung im direkten Bild gehalten und haben keine genaueren Bestimmungen im Nachbilde mehr versucht. 
410 J. K. A. Wertheim Salomonson and G. J. Schoute:

Fig 3
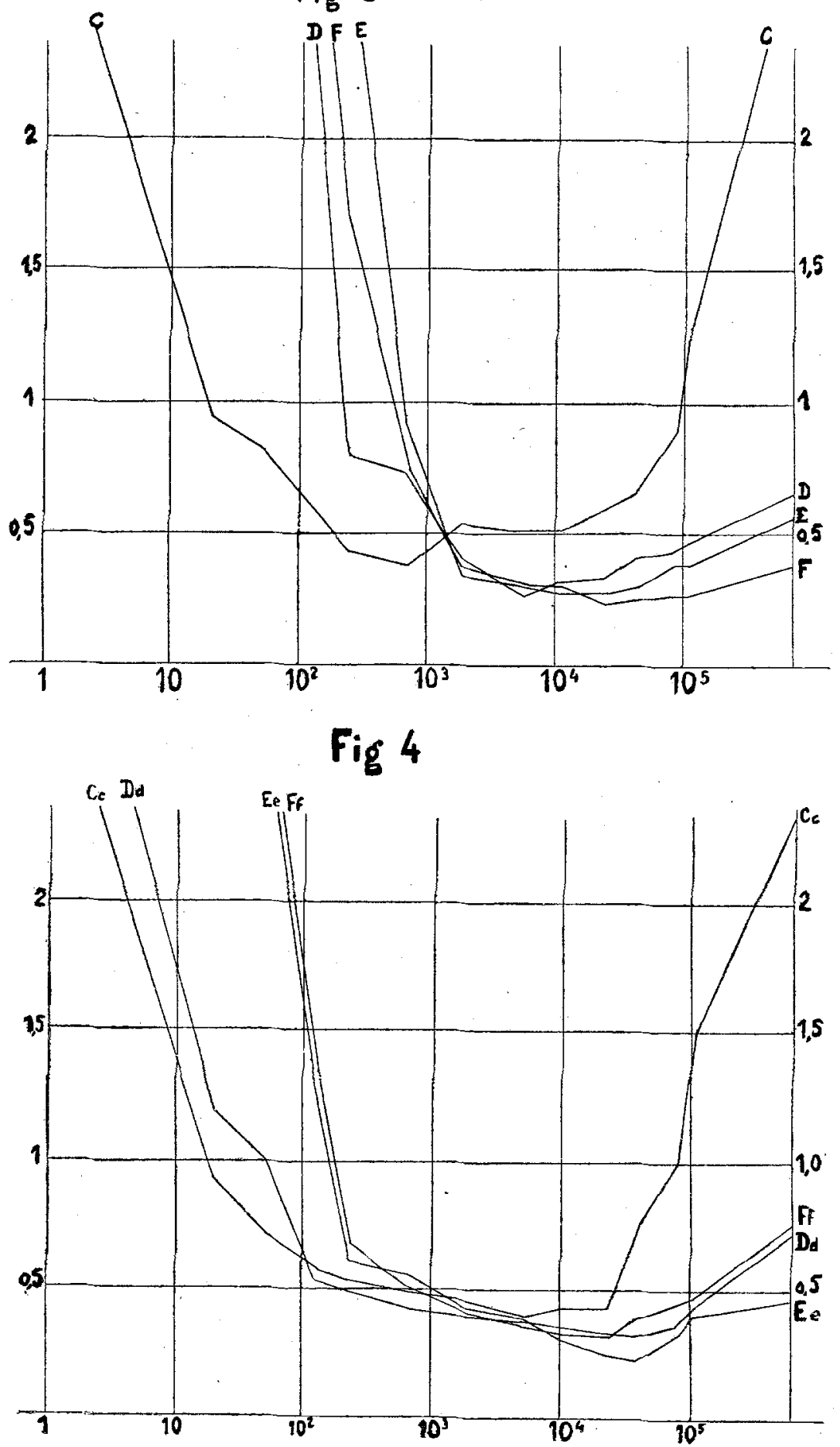
In zweiter Linie ergab sich, dass die konvexen Linien, die wir nun erhielten (siehe Fig. 3 und 4) unter sich ungefähr wieder kongruent und nur auf der Abscissenachse gegeneinander etwas verschoben waren.

Weiter zeigte es sich, dass im Vergleich mit der Krümmung der Kurven in Fig. 1 und 2 die Biegung bedeutend zugenommen hatte. Sowohl das Sinken der Kurve wie auch das spätere Steigen geschahen viel schneller, während der Höhepunkt des maximalen Unterscheidungsvermögens nur verhältnismässig wenig gestiegen war.

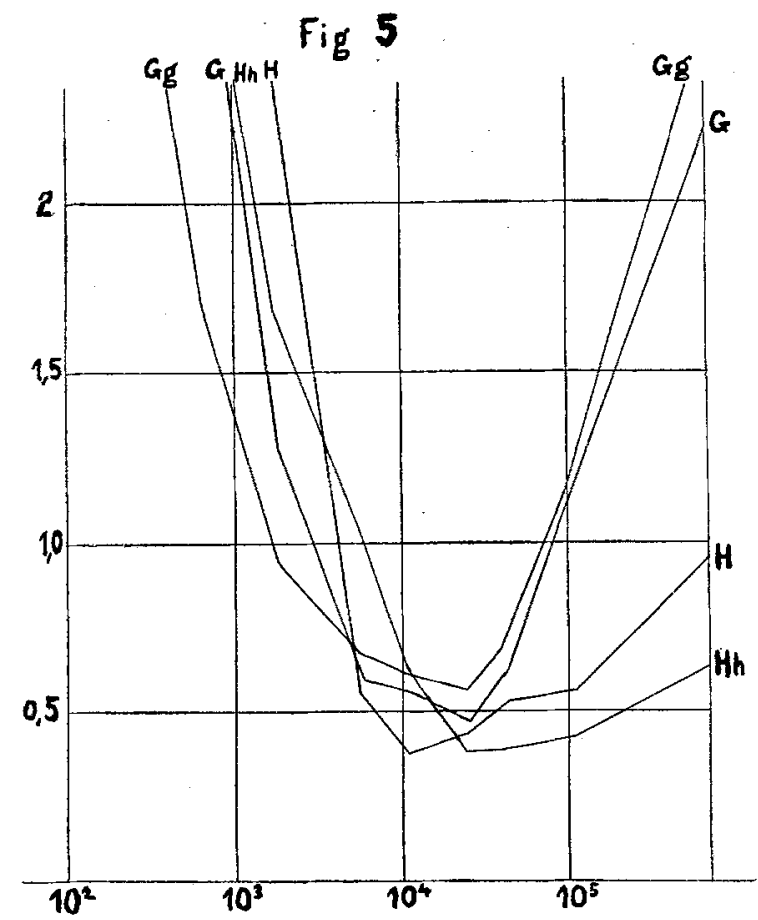

Bei Adaptation an grössere Lichtstärke scheint der minimale Schwellenwert jedoch geringer zu 'sein als bei Adaptation an die Dunkelheit oder an geringere Lichtstärke. Dies konnte vielleicht seine Erklärung in dem Umstande finden, dass die Wahrnehmungen in einem absolut dunklen Zimmer aussergewöhnlich schwierig waren, während bei einer Adaptation an eine Lichtstärke von 5,5 M.-K. oder an eine böhere Lichtstärke nicht die geringste Schwierigkeit vorhanden war. 

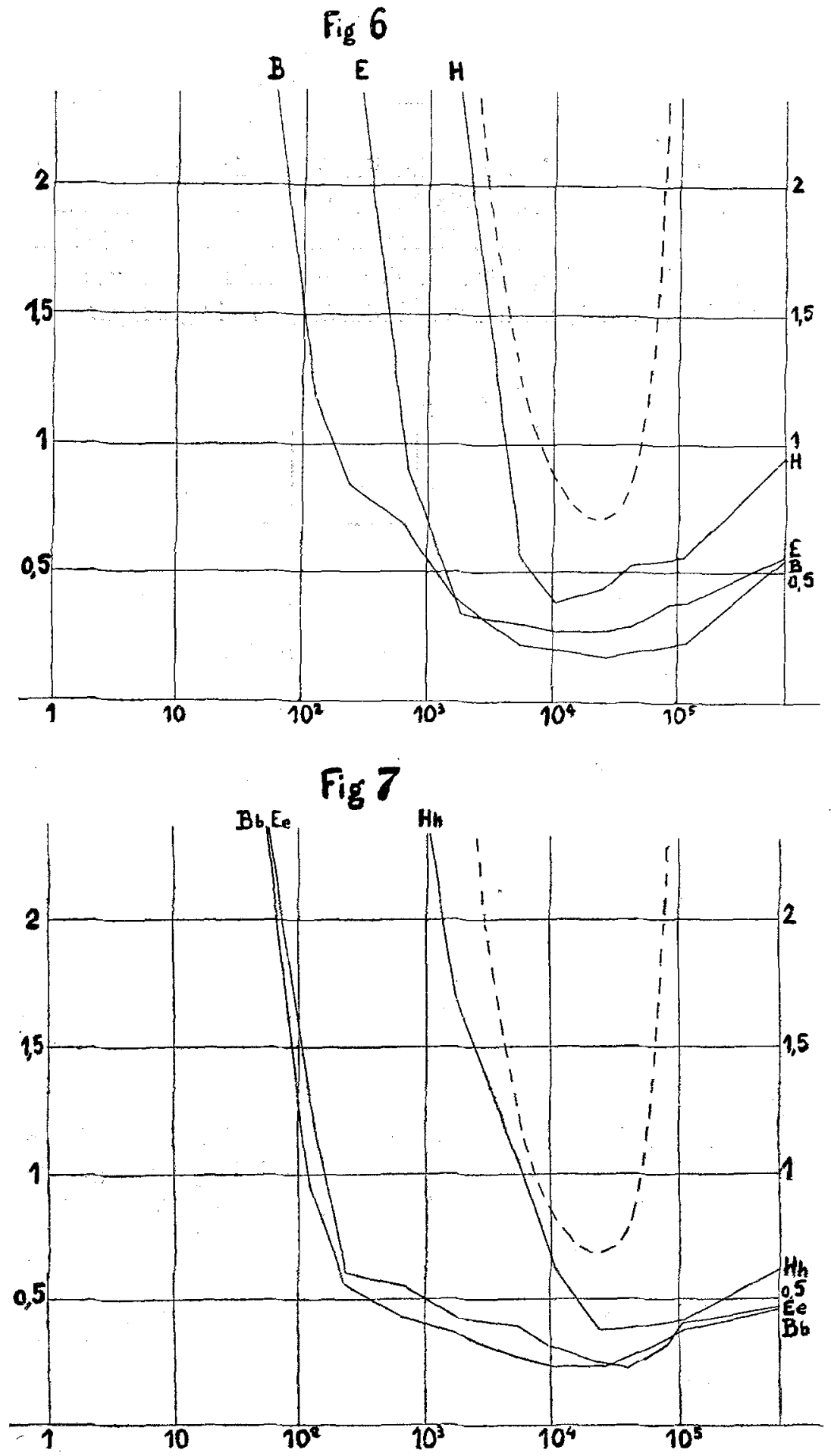
Interessant schien indessen die Tatsache, dass die Verkleinerung der den Reiz ausübenden beleuchteten Fläche die Kurve schneller sinken und steigen machte. Wir trachteten deshalb, auf diesem Wege noch weiterzugehen und verkleinerten den Winkel, unter dem die beleuchtete Fläche gesehen wurde, noch $21 / 2 \mathrm{mal}$, indem wir vor das Auge ein Galiläi'sches Fernrohr brachten, dessen Objektiv dem Auge, dessen Okular der beleuchteten Fläche zugekehrt war. Eine Reihe von Versuchen, die gemacht wurden bei einem Adaptationszustand an Lichtstärken von 5,5 und 50 M.-K., lieferte uns die Serien $G, G g, K, K k$, welche in Fig. 5 wiedergegeben sind.

Um den Einfluss zu zeigen, den die Verkleinerung der Lichtquelle auf den Lauf der Kurve hat, werden in Fig. 6 und 7 die Kurven $B, E, H$ und $B b, E e, H h$ gegeben, die sprechender, als Zahlen dies vermögen, uns zeigen, wie die Kurve fortwährend schmaler und steiler wird, je mehr die Bildgrösse auf der Retina abnimmt.

In derselben Figur ist noch eine punktierte Kurve wiedergegeben, die nach der Formel $\varrho=k \frac{e^{\beta R}}{R}$ konstruiert wurde. Ein Blick lebrt, dass die Linie, die wir für den Schwellenwert bei verschiedenen Lichtstärken erhalten, sich in dem Masse dieser theoretischen Kurve nähert, in dem die Lichtquelle kleiner wird, je weniger also peripherische Netzhautelemente gereizt werden.

Diese Tatsache musste uns auf die Vermutung bringen, es könne eine noch grössere Annäherung an die theoretische Kurve herbeigeführt werden, wenn wir die Grösse des angewandten Lichtreizes dermassen verminderten, dass nur ein einzelner Netzhautzapfen gereizt würde.

Bevor wir jedoch an die Untersuchung dieses Punktes gingen, suchten wir erst noch festzustellen, inwieweit eine Veränderung in der Wabrnehmungszeit von Einfluss auf die Kurve der Unterschiedsschwelle sei. Aus verschiedenen Gründen glaubten wir, dass uns eine Verkürzung der Wahrnehmungszeit weniger Vorteil bieten würde als eine Verlängerung. Wir haben jedoch dabei nicht getrachtet, ein vollständiges und genaues Bild zu erhalten, sondern nur eine Andeutung.

Diese konnte leicht erhalten werden aus einer neuen Reihe von Versuchen mit verlängerter Wahrnehmungszeit, in casu 1/25 Sekunde. Wir beschränlten uns überdies auf einen einzigen Adaptations- 

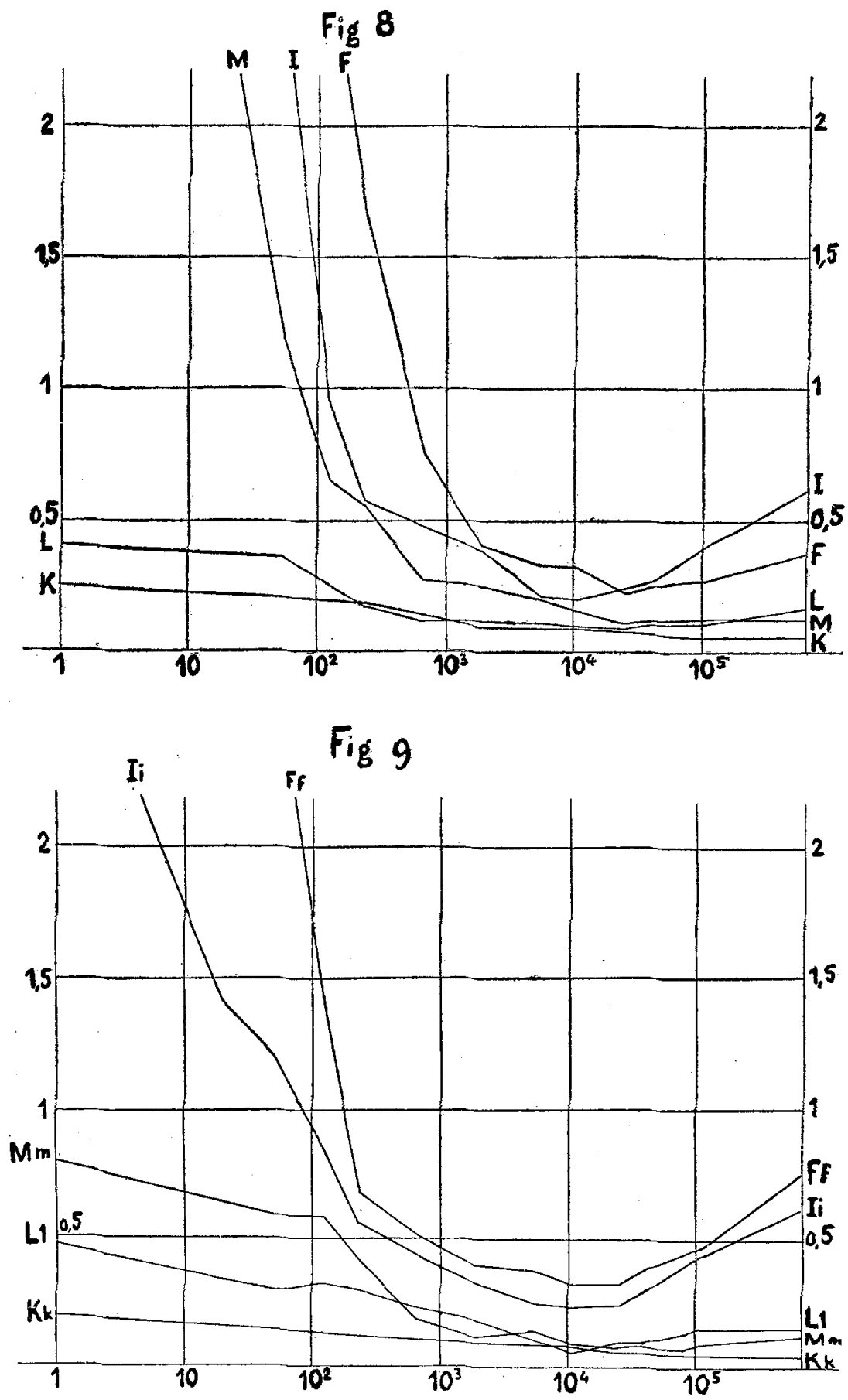
zustand, nämlich an eine Lichtstärke von 350 M.-K. Diese Serien sind zusammengefasst in die Zahlenreihen $I$ und $I i$ und graphisch wiedergegeben in Fig. 8 und 9. In dieser Figur haben zugleich die Reihen $F$ und $F f$ ihre Stelle gefunden.

Ein einziger Blick auf die Figur lehrt sogleich, dass die Verlängerung der Wahrnehmungzeit zur Abplattung der Kurve führt.

In derselben Figur sind ausserdem noch einige Kurven wiedergegeben, die bei einer längeren Wahrnehmungszeit erhalten wurden, und zwar Kurve $M$ und $M m$ und $L$ und $L l$ bei einer Wahrnehmungszeit von einigen Sekunden, während bei $K$ und $K k$ diese Zeit stets mehrere Minuten betrug und dabei eine möglichst vollständige Adaptation an die als Reiz dienende Lichtstärke erreicht worden war.

Wir sehen also, dass in dem Masse, in dem die Adaptation vollkommener ist, auch eine Annäherung an die gerade Linie des Weber-Fechner'schen Gesetzes mehr und mehr zutage tritt, vor allem im Gebiete der geringeren Lichtstärken.

\section{Erregung eines einzelnen Netzhautzapfens.}

Wir wiesen soeben schon darauf hin, dass die Kurve der Unterschiedsschwelle sich um so mehr der theoretischen Kurve $\varrho=k \frac{e^{\beta R}}{R}(3)$ nähere, je mehr die Anzahl der in Erregung gesetzten Netzhautelemente abnahm. Es lag also auf der Hand, Reizungsversuche mit nur einem Netzhautzapfen vorzunehmen. Nach Art der Sache war dies auf die bisher geübte Weise nicht möglich. Denn es würde ja, wenn wir das runde Feld so sehr verkleinerten, dass nur ein Netzhautzapfen getroffen worden wäre, kein Unterschied zwiscben den beiden Lichthälften mehr wahrnehmbar gewesen sein. Wir waren daher genötigt, unsere Versuche auf ganz andere Weise einzurichten, und wählten dazu die folgende

\section{Einrichtung für unsere Versuche.}

Fine geschlossene bölzerne Kiste von ungefähr $50 \mathrm{~cm}$ Höhe, $30 \mathrm{~cm}$ Breite und $40 \mathrm{~cm}$ Länge hat in einer Wand eine Öffnung von $6 \mathrm{~cm}$ Durchmesser, welche von einer kuglig gewölbten Milchglasplatte von ungefähr $8 \mathrm{~cm}$ Radius abgeschlossen wird. Im Zentrum der Linie befindet sich eine Glühlampe von 25 Kerzen Leuchtkraft, durch welche eine vollkommen gleichmässig beleuchtete Fläche er- 
halten wird. Vor dieser Milchglasplatte ist eine Metallplatte mit zwei Öffnungen von je $6 \mathrm{~mm}$ Durchmesser angebracht; die eine Öfnuug ist von der anderen $5 \mathrm{~cm}$ entfernt. Vor jeder der beiden Öffnungen befindet sich eine drehbare Scheibe mit kleineren Öffnungen. Die eine der drehbaren Scheiben hat deren zwölf, von $2^{1 / 4}$ bis $5 \mathrm{~mm}$ Durchmesser, die folgende Öffnung immer $1 / 4 \mathrm{~mm}$ grösseren Durchmesser als die vorhergehende. Die vor der anderen Öffnung angebrachte drehbare Scheibe zählt fünf Öffnungen, und zwar von $3,3^{1 / 4}, 3^{1 / 2}, 3^{3 / 4}$ und $4 \mathrm{~mm}$ Durchmesser. Vor dieser Vorrichtung, die um $180^{\circ}$ gedreht werden kann, so dass die rechte Scheibe die linke und umgekehrt die linke die rechte werden kann, sind einige Zentimeter entfernt zwei parallel laufende Leisten mit Nuten angebracht, in welche die Rauchgläser $1-8$ und a-i eingeschoben werden können. Unmittelbar davor befindet sich ein Momentverschluss (Tachistoskop).

Die Versuchsperson sitzt $10 \mathrm{~m}$ vom Apparate entfernt in einem vollständig dunklen Zimmer. Der Experimentator hat seinen Platz neben dem Apparat in der daranstossenden Dunkelkammer. Diese ist von einer Glühlampe von acht Kerzen Leuchtkraft, die noch mit zwei Lagen Papier umwickelt ist, spärlich beleuchtet: die Beleuchtung ist für die Versuchsperson gerade hinreichend, um den Ort, an dem beim Öffnen des Verschlusses die beiden beleuchteten Öffnungen sichtbar werden, zu fixieren.

Alle Versuche wurden einige Stunden nach Sonnenuntergang vorgenommen, die Übungsserien zuerst bei einer Entfernung von $10 \mathrm{~m}$, einige Serien danach bei $4 \mathrm{~m}$ Entfernung, die definitiven Serien schliesslich wieder bei $10 \mathrm{~m}$ Entfernung.

Der Experimentator dreht die eine der drehbaren Scheiben so, dass eine ihrer Öffnungen - meistens war es die von 3 oder von $3^{1 / 2} \mathrm{~mm}-$ vor die eine Öffnung der dahinter befindlichen Metallscheibe $\mathrm{zu}$ stehen kommt und eine willkürlich gewählte Öffnung der anderen Drehscheibe vor die zweite Öffnung in der Metallplatte.

Nachdem das Tachistoskop gespannt worden ist, fixiert die Versuchsperson. Sie ruft alsdann „ja“, und unmittelbar danach öffnet der Experimentator den Verschluss. Hierauf teilt die Versuchsperson mit, welche Öffnung ihr heller erschienen ist, die recbte oder die linke. Der Versuch wird so lange wiederholt, bis endlich die Unterschiedsschwelle erreicht ist. 
Da die Ermüdung, welche derartige Versuche im Gefolge hat, verhältnismässig schnell eintritt, so wurde die Anzahl Beleuchtungen für jeden Punkt der Kurye in der Regel auf 15-20 beschränkt, während wir nicht mehr als 6 oder 7 Punkte bestimmten. Jede Serie bestand aus ca. 100 bis 120 einzelnen Wahrnehmungen. Im ganzen wurden 26 Serien bestimmt (von jedem von uns 13).

Die Schnelligkeit des Verschlusses betrug bei der ersten Versuchsreihe 1/25 Sekunde (genauer 0,0407"): es zeigte sich jedoch alsbald, dass bei dieser Schnelligkeit die Beobachtungen ungenau wurden. Wir erhöhten darum sehr bald die Zeit auf 0,1 Sekunde (genauer 0,0944").

In Tabelle III sind die Resultate einiger Serien mitgeteilt, so, wie sie protokolliert wurden.

Tabelle III.

\begin{tabular}{|c|c|c|c|c|}
\hline Glas & W. S. N. & Sch. Nn. & W. S. 0. & Sch. Oo. \\
\hline $\begin{array}{c}0 \\
b \\
6 \\
b+6 \\
4+d \\
5+7 \\
i \\
i+a \\
i+5 \\
i+b\end{array}$ & $\begin{array}{l}5 \\
4^{3 / 4} \geq 3 \\
4^{3 / 4} \geq 3 \\
4^{2 / 4} \geq 3 \\
3^{3 / 4}>3 \\
3^{1 / 2} \geq 3 \\
3^{3 / 4}>3 \\
4^{1 / 4} \geq 3 \\
4^{2 / 4}>3 \\
\text { unsichtbar }\end{array}$ & $\begin{array}{l}5 \\
4^{3 / 4} \geqq 3 \\
4^{2 / 4} \geq 3 \\
4^{1 / 4}>3 ; 4 ? \\
4 \text { aे } 4^{1 / 4} \geq 3 \\
4 \quad \geq 3 \\
44^{1 / 4} \geq 3 \\
4^{1 / 2}>3 \\
4^{3 / 4} \geq 3 ; 4^{2 / 4} \geqq \\
4^{3 / 4}>3\end{array}$ & $\begin{array}{l}5>3 \\
4^{3 / 4} \geq 3 \\
4^{2 / 4} \geq 3 \\
41 / 4>3 \\
4^{1 / 4} \geq 3 \\
4 \geq 3 \\
4 \geq 3 \\
4^{2 / 4} \geq 3 \\
4^{3 / 4}>3 \\
\text { unsichtbar }\end{array}$ & $\begin{array}{l}4^{3 / 4}>3 \\
4^{1 / 4} \geq 3 \\
4^{1 / 4}>3 \\
4^{1} / 4 \geq 3 \\
4 \quad>3 \\
4 \geq 3 \\
3^{3} / 4 \geq 3 \\
4 \quad \geq 3 \\
4 \quad>3 \\
\text { unsichtbar }\end{array}$ \\
\hline
\end{tabular}

Bei diesen Versuchen wurde die Lichtquelle durch Vergrösserung oder Verkleinerung verändert.

Von H. A. La a ${ }^{1}$ ) und einem der Verfasser ${ }^{2}$ ) ist nachgewiesen worden, dass dies eine tatsächlich erlaubte Methode ist, wenn wir es mit einer Lichtquelle von genügend geringer Grösse zu tun haben, oder, besser gesagt, wenn unser Retinabild klein genug ist. In diesem spezjellen Falle haben wir danach gestrebt, ein Lichtbild zu erhalten, das ungefähr einen Netzhautzapfen bedeckte. Wenn das Bild jeder der beiden Öffnungen mit den Grössen des schematischen Auges

1) H. A. Laan, Over gezichtsscherpte en hare bepaling. Inaug.-Disserts Utrecht 1901.

2) G. J. Sch oute, Waarnemingen met een enkelen Netvlieskegel. Inaug. Dissert. Leiden 1898. 
Listings berechnet wird, ergibt sich eine 10000/15malige Verkleinerung, und betrug es also nur 0,0045-0,0075 mm.

Da der Durchschnitt eines Netzhautzapfens ungefähr $0,0044 \mathrm{~mm}$ beträgt ${ }^{1}$ ), dürfen wir annehmen, dass in der Tat nicht mehr als höchstens 1-3 Kegel getroffen wurden.

Um unsere Reihen berechnen zu können, war es notwendig, die Grösse einer jeden Diaphragmaöffnung sehr genau zu kennen, wir durften uns nicht auf den Instrumentenmacher verlassen: Die Messung fand statt, indem mit Hilfe eines Zeiss' schen Projektionssystems ein etwa 30 mal vergrössertes Bild auf eine Mattglasscheibe projiziert wurde. Dieses Bild wurde in vier Meridianen genau gemessen und hieraus der Durchschnitt genommen als Grundlage für die Berechnung der Oberfläche. Wir fanden nun in bezug auf das Verhältnis der verschiedenen Oberflächen

$\begin{array}{ccc}\text { Öffnungen } & \text { Grosse Scheibe } & \text { Kleine Scheibe } \\ 2^{1 / 4} & 0,558 & - \\ 2^{2 / 4} & 0,654 & - \\ 2^{3 / 4} & 0,755 & - \\ 3 & 0,921 & 1,000 \\ 3^{1 / 4} & 0,994 & 1,083 \\ 3^{2 / 4} & 1,151 & 1,264 \\ 3^{2 / 4} & 1,469 & 1,434 \\ 4 & 1,760 & 1,686 \\ 4^{1 / 4} & 1,899 & - \\ 4^{2 / 4} & 2,162 & - \\ 4^{3 / 4} & 2,463 & - \\ 5 & 2,742 & -\end{array}$

wobei die kleinste Öffnung der kleinen Scheibe als Einheit angenommen wurde.

Die Veränderung, die jede folgende Öffnung in der Lichtstärke zustande brachte, war sehr bedeutend. Der Gebrauch des Apparates lehrte indessen bald, dass diese Sprünge in Wirklichkeit nicht zu gross waren, und dass eine feinere Gradation der öffnungen die Schwierigkeit nur erhöht hätte, ohne dass sie der Genauigkeit der Bestimmungen zugute gekommen wäre.

Bei unseren Versuchen zeigte es sich, dass wir nicht immer eine Unterschiedsempfindung von gleicher Qualität erhielten: einmal

1) W. Koster Gzn., Bijdrage tot de Kennis der fovea centralis retinae Nederl. Tijdschr. v. Gen. t. 2 p. 358.1895. 
gewannen wir den Eindruck, der eine Fleck sei heller als der andere, dann wieder, dieser Fleck sei grö össer als de andere.

Der Aspekt war bei kleineren Lichtstärken stets der eines leuchtenden Punktes; bei weniger absorbierenden Gläsern oder ohne Gläser erhielten wir stets den Eindruck eines sternförmigen Fleckes; dieser Eindruck wurde gewöhnlich schon hervorgerufen bei Gebrauch von Glas 6 oder $\mathrm{b}+6$ und bei sämtlichen helleren Platten.

\section{Resultat der Versuche.}

Wir haben so viel wie möglich getrachtet, eine genaue Übersicht über die Veränderung der Unterschiedsschwelle bei verschiedenen Lichtstärken jzu gewinnen, und das Resultat unserer Untersuchungen gibt nachstehende Tabelle.

$\begin{array}{cc}\text { Lichtstärke } & \begin{array}{c}\text { Unterschieds- } \\ \text { schwelle }\end{array} \\ 1,00 & >2,40 \\ 2,94 & 1,32 \\ 4,43 & 0,96 \\ 6,03 & 0,62 \\ 11,9 & 0,54 \\ 24,7 & 0,60 \\ 43,2 & 0,86 \\ 88,6 & 1,21 \\ 185,4 & 1,43 \\ 380,0 & 1,60\end{array}$

In Fig. 10 ist diese Tabelle graphisch dargestellt.

Wir geben hierbei zugleich eine punktierte Linie, welche die Richtung der theoretischen Kurve darstellt, entsprechend der Formel $\varrho=K \frac{\varepsilon^{\beta R}}{R}$.

\section{Fig 10}

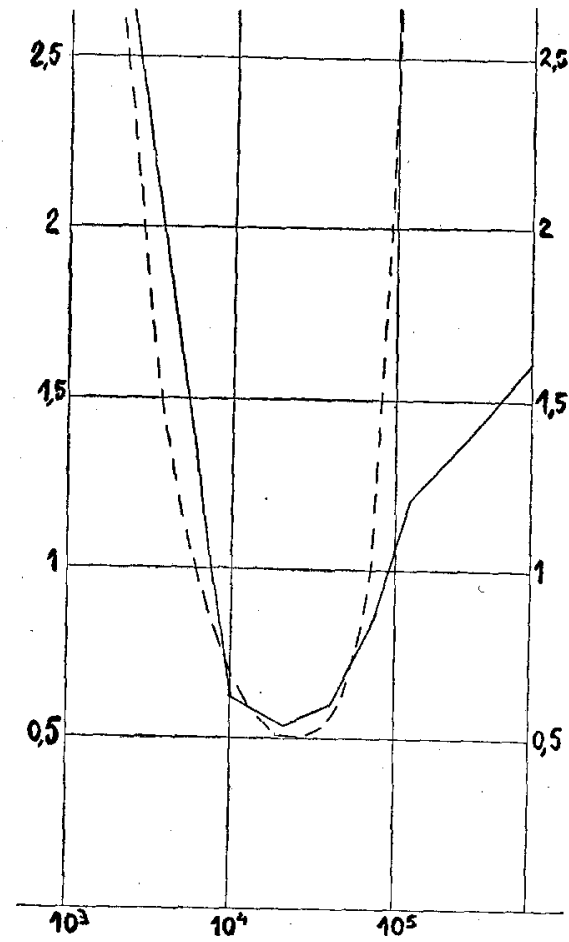

Nun zeigt es sich, dass in der Tat eine ziemlich grosse Übereinstimmung besteht, und zwar bis zur Lichtstärke 88,6. Von hier ab steigt die auf experimentellem Wege gefundene Kurve weniger schnell als die theoretische Kurve. 
Von Wichtigkeit ist es, hier gleich zu bemerken, dass an demselben Punkte der Kurve sich der Einfluss der Irradiation bemerkbar macht.

Nun ist von einem der Verfasser ${ }^{1}$ ) darauf hingewiesen, dass angenommen, das Reizungsgesetz gelte in der Tat für momentane Reize auf einzelne wenigen Endorgane - in jedem Falle dann durch Irradiation eine Korrektur in der Kurve der Unterschiedsschwellenwerte bewirkt werden wird, wodurch sich die Kurve einigermassen dem Gesetze Weber-Fechner's nähert.

Im vorbergehenden haben wir gezeigt, dass verschiedene Umstände vorbanden sind, die die Ursache werden, dass eine Kurve der Unterschiedsschwellenwerte nicht stets dieselbe Form zeigt. Wenn es möglich wäre, ein einzelnes Neuron mit vollkommenem Ausschluss aller anderen benachbarten Neuronen isoliert zu reizen, dann würden wir eine Kurve erwarten können, die gänzlich mit unserer theoretischen Kurve (2) übereinstimmte.

Der Umstand, dass das Auge allein mittelst Zerstreuungskreisen sieht, bewirkt, dass bei böheren Lichtstärken immer Irradiationserscheinungen auftreten werden.

Wir nehmen also in der Irradation eine der ersten Korrekturen des Reizungsgesetzes in der Richtung des Weber-Fechner'schen Gesetzes an.

Eine zweite Korrektur haben wir bei unseren ersten Versuchsreihen in der Bildung des Nachbildes kennen gelernt.

Ohne auf die physiologische Genesis des Nachbildes näher einzugehen, dürfen wir doch sagen, dass wir in dem Nachbild ein physiologisches Hilfsmittel haben kennen gelernt, dass imstande ist, die Kurve der Unterschiedsschwellenwerte einer horizontalen Linie, welche parallel zur Abscissenachse läuft, zu nähern.

Die dritte Korrektur ist gegeben in dem Umfang des Reizes. In dem Masse, in dem der Reiz extensiver wird, also eine grössere Anzahl Neuronen trifft, in dem Masse wird die Kurve der Unterschiedsschwellenwerte flacher werden und sich mehr der Horizontallinie nähern.

Die vierte und fünfte Korrektur liegt in der Dauer des Reizes.

Die Korrektur, welche von der Zeit bewirkt wird, ist nämlich eine zweifache. Schon die Tatsache allein, dass der Reiz von längerer Dauer ist, macht die Kurve flacher.

1) J. Wertheim Salomonson, Verslag d. Koninkl. Akad. v. Wetenschappen. 25. Februar 1902. Siehe auch Anhang II. 
Daneben jedoch ist noch ein zweiter Einfluss der Zeit vorhanden, nämlich der Einfluss auf den Adaptationszustand des Organs: je länger ein Reiz dauert, desto mehr wird das gereizte Organ geeignet, geringere Veränderungen in diesem Reize zu perzipieren (innerhalb bestimmter Grenzen).

Hieraus ergibt sich ferner, dass wir als Bedingung für die Gültigkeit des Weber-Fechner'schen Gesetzes stellen können:

1. der Reiz muss eine genügend grosse Anzahl Endorgane treffen;

2. der Reiz muss von genügend langer Dauer sein.

Zum Schlusse noch die Bemerkung, dass unsere Schlüsse gezogen sind aus Beobachtungen über das Gesichtsorgan und allein auf dieses Organ anwendbar sind. Es stehen uns bis jetzt noch keine Daten zu Dienste, die uns das Recht gäben zu generalisieren.

\section{Anhang $I$.}

Es sei nach dem Reizgesetze (I) der Effekt $E$ von einem peripherischen Reize $R$ auf ein Teleneuron verursacht:

$$
E_{1}=A_{1}\left\{1-\varepsilon^{-B_{1}\left(R-C_{1}\right)}\right\} \quad . . .
$$

oder auch

$$
E_{1}=A_{1}-c_{1} \varepsilon^{-B_{1} R} \quad \text {. . . . . }
$$

wenn wir $c=\mathrm{A}_{1} \varepsilon^{B_{2} C_{1}}$ stellen.

Denken wir uns, dass dieser Effekt selbst wieder einen Reiz $R_{11}$ für ein Deuteroneuron bildet, dann ist der am Deuteroneuron beobachtete Effekt:

und da $R_{11}=E_{1}$ :

$$
E_{11}=A_{11}-c_{11} \varepsilon^{-B_{11} R_{11}}
$$

$$
E_{11}=A_{11}-c_{11} \varepsilon^{-B_{11}\left\{A_{1}-c_{1} \varepsilon^{-B_{1} R}\right\}} . . .
$$

Substituieren wir $c=c_{11} \varepsilon^{-B_{11} A_{1}}$, dann wird:

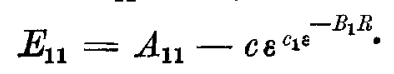

Hieraus erhalten wir durch Reihenentwicklung:

$$
\begin{array}{r}
E_{11}=A_{11}-c\left\{1+c_{1} \varepsilon^{-B_{1} R}+1 / 2 c_{1}^{2} \varepsilon^{-2 B_{1} R}+\right. \\
\left.1 / 6 c_{1}^{2} \varepsilon^{-3 B_{1} R}+\text { usw. }\right\} . . . \text {. (IV). }
\end{array}
$$

Werden die höheren Glieder dieser Reize vernachlässigt, und beschränken wir uns auf die zwei ersten Glieder, dann erhalten wir:

$$
E_{11}=A_{11}-c-c c_{1} \varepsilon^{-B_{1} R}
$$


oder auch

wobei $\alpha=A_{11}-c$, und $\gamma=c c_{1}$.

$$
E_{11}=\alpha-\gamma \varepsilon^{-B_{1} R} \text {. . . . . . (V), }
$$

Diese Formel ist identisch mit (II).

War es aber erlaubt, die höheren Glieder der Reihe in IV zu eliminieren? Nach allem, was wir von der Reizübertragung als Nervenfunktion wissen, ist diese Frage unbedingt zu bejahen. Ich branche ausserdem nur hinzuweisen auf meine frühere Arbeit in diesem Archiv Bd. 100 S. 470. Es wurde daselbst eine Versuchsreihe Waller's mitgeteilt, aus der unmittelbar hervorgeht, dass die Nervenleitung durch eine lineäre Funktion ersten Grades ausgedrückt werden kann, und dass somit eine Beschränkung auf zwei Glieder statthaft ist.

Da bei einer eventuellen Übertragung des Reizes auf ein drittes odier viertes oder noch höheres Neuron immer die nämliche Überlegung gilt, können wir anstandslos voraussetzen, dass auch für die Reizübertragung auf böhere Neuronen, d. b. bei psychischen Prozessen, unter bestimmten Bedingungen zwischen bestimmten Grenzen ein Zutreffen meines Reizgesetzes erwartet werden dürfte.

\section{Anhang II.}

Wenn es wirklich möglich wäre, ein peripherisches Neuron vollständig isoliert zu reizen, dürfte eine befriedigende Ubereinstimmung mit dem Reizgesetz zu erwarten sein. Allein eine absolute Beschränkung des Reizes auf ein einziges Neuron ist undenkbar, und somit ist also voraussichtlich eine Abweichung dieses Gesetzes zu erwarten. Dies geht unmittelbar aus folgenden Betrachtungen hervor.

Nehmen wir als Beispiel den Versuch, eine sehr geringe Zahl Endorgane in der Haut durch Druck mit einem spitzigen Gegenstand zu reizen. Auf sämtlichen unmittelbar unter dem Objekt - mit dem wir den Druck ausfübren - liegenden Endorganen wird dabei ein gleichmässiger Lruck ausgeübt, falls wir die Haut als vollkommen elastisch annehmen. Wird der Druck grösser, so nimmt proportional der Reiz zu; für die Endorgane $a, a, a, a$ wird also unser Gesetz gelten. Wenn der Druck zunimmt, ändert aber die Hautoberfläche ihre Form und wird teilweise komprimiert. Hierbei werden aber auch Endorgane $b, b$, welche ausserhalb der ursprüng- 
lich komprimierten Region liegen, zusammengedrückt werden. Bei noch grösserem Drucke werden sogar entferntere Endorgane $c, c, d, d$ gereizt werden. Von allen diesen Endorganen werden dem Zentralnervensystem Impulse zugehen, dessen Gesamteffekt unser Urteil bestimmt.

Die Neuronen $b, b, c, c, d, d$ gehorchen zwar dabei ebenfalls dem Reizgesetze, aber die Reizintensität ist für die verschiedenen Neuronen auch verschieden. Es gelten somit für diese Neuronen die Ausdrücke:

$$
\varrho_{1}=k \frac{\varepsilon^{B r_{1}}}{r_{1}}, \varrho_{2}=k \frac{\varepsilon^{B r_{2}}}{r_{2}}, \varrho_{3}=k \frac{\varepsilon^{B r_{3}}}{r_{3}} \text { usw. }
$$

Da. die Einzelreize $r_{1}, r_{2}, r_{3}$ dem Gesamtreize $R$ proportional sind, dürfen wir diese stellen zu: $m_{1} R, m_{2} R, m_{3} R$ u. s. w.

Jetzt entsteht die Frage: Wie werden wir psychisch die verschiedenen Impulse verarbeiten, damit ein bestimmter Zweck Vergleichung zweier um ein weniges verschiedener Reize - am zweckmässigsten erreicht wird? Einfache Addition oder Reizsummation ist dabei ausgeschlossen: dies würde in Widerspruch stehen mit unserer Erfahrung, dass durch Fixierung der Aufmerksamkeit auf eine bestimmte Empfindung jede andere Empfindung geschwächt wird. Wir werden also in diesem Falle nicht den Gesamteindruck für unsere Bestimmung verwenden, sondern nur denjenigen Teil desselben, der sich am besten dazu eignet.

Von diesem Standpunkte aus können wir der Frage mathematisch nähertreten.

Durch Vergrösserung des Reizes wird in unserem Falle ohne Ausnahme eine Vergrösserung der Zahl gereizter Neuronen erfolgen. Diese Vergrösserung darf als eine, nicht unerhebliche angesehen werden. Für jedes neue Neuron haben wir einen neuen Koeffizienten $m$. Konstruieren wir jetzt zahlreiche Kurven von $\varrho$ mit verschiedenen $m$, dann nehmen wir an, dass unser Urteil über eine Differenz zweier Reize vorzugsweise immer durch ein bestimmtes Stück einer bestimmiten Kurve, wiedergegeben wird, und zwar bei Änderung des Grundreizes auch durch Benutzung einer anderen Kurve aus der ganzen Kurvenschar. Mathematisch ausgedrückt, heisst dies, dass die einhüllende Kurve oder Enveloppe eine graphische Darstellung über die Empfindlichkeit unseres Urteils ergibt. 
424 J. K. A. Werth. Salomonson u. G. J. Schoute: Psychoop. Unters.

Die einhällende Kurve für die Kurvenschar $\varrho=K \frac{\varepsilon^{m B} R}{m R}$ bei veränderlichem $m$ wird berechnet, indem wir zuerst $F=\varrho-$ $K \frac{\varepsilon^{m B R}}{m R}$ stellen.

Jetzt berechnen wir den Wert von $m$, welcher sich aus $\frac{d F}{d m}=0$ ergibt; dieser wird in $F=0$ substituiert.

$$
\frac{d F}{d m}=-\frac{K}{R} \cdot \varepsilon^{m B R} \cdot \frac{m B R-1}{m^{2}}=0
$$

also $m=\frac{1}{B R}$, welehes substituiert in $F=0$ ergibt:

$$
\varrho=K B \varepsilon \text {. }
$$

$\varrho$ ist also konstant, $d$. h. die relative Unterschiedsschwelle ist konstant. Dies ist das Weber'sche Gesetz.

Aus dieser Betrachtung geht hervor, dass bei Reizung von sehr wenigen Neuronen unser Reizgesetz nur beschränkte Gültigkeit besitzt. Bei Vergrösserung des Reizes muss Irradiation des Reizes stattfinden, welche eine Abweichung des Reizgesetzes zur Folge hat und eine Annäherung an das W eber'sche Gesetz hervorruft.

\section{Anhang III.}

Aus $E=A\left(1-\varepsilon^{-B(R-c)}\right)$ bilden wir:

$$
\frac{d E}{d R}=A B \varepsilon^{-B(R-C)}
$$

und

$$
d R=\frac{1}{A B} \cdot \varepsilon^{B(R-c)} d E .
$$

Nehmen wir statt unendlich kleine $d F$ endliche, jedoch genügend kleine Differenzen $\triangle E$, dann ist noch statthaft

$$
\triangle R=\frac{1}{A B} \cdot \varepsilon^{B(R-c)} \triangle E \text {. }
$$

Nehmen wir mit Fechner an, dass $\triangle E$, eine eben merkliche Empfindungszunahme, als eine konstante Grösse betrachtet werden darf, dann ist $\triangle R=k \varepsilon^{B R}$.

Diese Formel gibt die Grösse der absoluten differentiellen Schwelle für jeden Reiz $R$.

Die relative Unterschiedsschwelle wird:

$$
\frac{\triangle R}{R}=\frac{k \varepsilon^{B R}}{R} \text {. }
$$

